\title{
Bericht über die 10. Versammlung der Deutschen otologischen Gesellschaft am 24. und 25. Mai 1901 zu Breslau.
}

\author{
Von
}

\author{
Dr. Edmund Wertheim in Breslau.
}

\section{Erste Sitzung, Freitag 24. Mai Vormittag's 10 Uhr.}

Der Vorsitzende, Herr Habermann-Graz, eröffnet die Sitzung mit einer Begrüssungsansprache, in der er an die Bedeutung Breslaus für die Entwicklung der Otologie unter Hinweis auf Middeldor f , Voltolini, Jacoby and Gottstein erinnert. Es folgt die Begrüssung der Versammlung Namens der Stadt Breslau durch Herrn Oberbürgermeister Dr. Bender und Herrn Stadtrath Dr. Steuer, welch' letzterer seines alten Freundes Dr. Ludwig Ja c ob y gedenkt, der, ein hochberziger Menschenfreund, sein ganzes, mühevoll erworbenes Vermögen der Stadt Breslau zur Errichtung einer Klinik für unbemittelte Ohrenkranke vermacht habe. Breslau sei die erste Stadt gewesen, die unter Zuhilfenahme communaler Mittel ein solches Institut geschaffen habe. Vor 10 Jahren, als dasselbe ins Leben trat, hätten in Dentschland nur 2 Universitäten ein solches besessen. Nachdem dann der Vorsitzende des Localcomités, Herr Kümmel, den städtischen Behörden für ihr Entgegenkommen und den Mitgliedern der Gesellschaft für ihr zahlreiches Erscheinen in Breslau gedankt hatte, constatirte der Vorsitzende mit Genugthuung die im verflossenen Geschäftsjahre erfolgte Ernennung des Herrn Körner zum Professor ordinarius an der Universität Rostock. Auf Vorschlag von Herrn Zaufal-Prag beschliesst die Versammlung dem Herzog Johann Albrecht von Mecklenburg-Schwerin durch den Ausschuss eine Dankadresse zu übermitteln. Der Vorsitzende gedenkt sodann des Ablebens Kuhn's Strassburg, des bei seinen Fachgenossen allgemein beliebten und angesehenen Mitbegründers der Gesellschaft, deren Ausschusse er bis zu seiner Erkrankung angehört habe. Die Versammlung ehrt sein Andenken durch Erheben von den Plätzen. Der Secretär verliest die Namen der $29 \mathrm{neu}$ a ufg en om menen Mit glieder, durch deren Eintritt die Mitgliederzahl der Gesellschaft auf 267 gestiegen ist. Nach erfolgtem Cassenberichte wurde dem Schatzmeister Entlastung ertheilt. Sodann beschliesst die Versammlung Herrn Kes s el - Jeua, der seinen Austritt aus dem Ausschusse schriftlich mitgetheilt hat, telegraphisch um Zurückziehung seiner Austrittserklärung zu ersuchen.

Hierauf beginnt die wissenschaftliche sitzung.

I. Herr Jansen-Berlin, Referat über: Der gegenwärtige Stand der Lehre von der otogenen Pyämie.

Trotz reichen Zuwachses zur Casuistik sind die Meinungsverschiedenheiten über die Pathologie der Sinusthrombose noch nicht beseitigt (Körner, Zaufal, v. Bergmann, Leutert). Zu unterscheiden sind 1) die obturirende septisch zerfallende Thrombose, 2) die wandständige Thrombose, 3) die Compressionsthrombose, die zur pyämischen Thrombose nicht führen kann, wenn der anliegende Herd operativ beseitigt wird. Ob extradurale $\mathbf{A b}$ scesse pyämische Metastasen hervorrufen kōnnen, ist fraglich. Eine vielumstrittene Frage ist ferner, ob pyämische Stoffe aus den Mittelohrräumen direct in die Cirkulation aufgenommen werden können. Der eitrige Process entfaltet durch die dünne vordere Sinuswand hindurch schneller seine Ein- 
wirkung, als durch die gesammte Dicke der Dura bindurch aufs Cerebrum. Man sieht deshalb bei extraduralen Abscessen, da sie nur auf das äussere Blatt der Dura einwirken, bei der Obduction ínen intacte Dura das Hirn umhüllen in Fällen, wo man annahm, dass die ganze Dura an jener Stelle gangränös sei. Bei Periphlebitis dagegen, besonders bei grossen, abgeschlossenen periphlebitischen Abscessen, findet eine Läsion des Endothels durcb septisches Material mit nachfolgender Thrombose statt. Die wandständige Thrombose kann ausheilen, wenn der äussere Herd beseitiot wird. Ein solider Thrombus von geringer Infectiosităt wird ausheilen nach frühzeitigem Ausheilen des Grundprocesses. Die Compressionsthrombose operativ anzugehen, ist ein Fehler; sogar die Berührung mit der Punctionsnadel kann eine Infection hineintragen. All' das Gesagte trifft auch für den Bul bus jugularis zu. Besonders bei Pyämieen durch acute Mittelohreiterung spielt die Bulbusthrombose eine grosse Rolle. In allen Fällen, wo bei Annahme von Sinusthrombose sich der Sinus normal erweist, muss man an Bulbustbrombose denken. Ausser Sinusphlebitis und Bulbusthrombose wird die im Innern des Warzenfortsatzes localisirte Eiterung selbst als Ursache der Pyämie beschuldigt. Von der Osteophlebitis darf man kaum einen anderen Effect erwarten, als von der Erkrankung des Knochens selber. Die osteophlebitische Pyämie ist aber von einigen Autoren, besonders $\mathrm{K} \ddot{\mathrm{r} n} \mathrm{n} \mathrm{r}$ als ein besonderes Krankheitsbild aufgestellt worden. Bezüglich der Frage der Spontanheilung von Sinusthrombose erklärt Jansen, dass er früher die geheilten Fälle vielfach als vom Processus mast. aus entstanden betrachtet habe, dass er aber, seitdem er systematisch den Sinus aufsuche und event. punctire, zu anderer Auffassung gekornmen sei, und in ibr durch Leutert's Erörterungen bestärkt wärde. Jansen hat bei Pyämie stets $S$ in usphlebitis constatirt. Er führt aus, dass das Weiterbestehen pyämischer Erscheinungen (hohen Fiebers, Schüttelfröste etc.) nach Ablauf' des Anfangsstadiums der Otitis media acuta purulenta die Annahme einer Sinnsphlebitis nahelege. Bei Säuglingen kann zwar, wie Ponfick und Brieger festgestellt haben, durch Aufnahme von Toxinen ins Blut Pyämie entstehen, bei Erwachsenen scheint das indes kaum vorzakommen. Jansen lengnet $\mathrm{zwar}$ nicht die Möglichkeit der Entstehung leichter pyämischer Zustände vom Eiterungsprocesse im Warzenfortsatze aus, bestreitet aber deren ofteres Vorkommen, practische Bedentung und vor allem Fähigkeit der Metastasenbildung. Für die Beurtheilung der Frage sind nur die Ergebnisse solcher Obductionen verwerthbar, bei denen alle Sinus und der Bulbus jugularis untersucht wurden. Kleine Thromben werden sogar manchmal erst nach Herausuahme des Präparates aus dem Schädel entdeckt. Alle Fälle von Osteophlebitis und perisinuösem Abscess baben für die Beurtheilung auszuscheiden. Jansen hat 60 Fälle von angeblicher Pyämie obne Sinusthrombose aus der Literatur gesammelt. Ohne Operatien sind 16 dieser Fälle geheilt, beì denen der Speculation freier Spielraum gelassen sei. Unter den 26 operirten Fällen ist die Operation in 15 Fällen ungenügend beschrieben oder ausgefïhrt; in sechs dieser operirten Fälle fand sich gesunder sklerosirter Knochen. Woher soll in letzteren Fällen die Osteophlebitis kommen? Periphlebitis dagegen kommt nach Jan s e $n$ bei negativem Befunde in Warzenfortsatze vor. Das Verhalten des Bulbus jugularis ist in keinem der 60 Fälle ausdrücklich erwähnt. Es bleibt also für die Betrachtung nur ein sehr kleiner Rest von Fällen, der seinerseits auch keine allzustrenge Kritik vertrage. In $25 \%$ dieser Fälle bestand chronische Mittelohreiterung, $25 \%$ sind gestorben, in ca. $25 \%$ bestandea Lungenmetastasen, Unter 63 ven Jansen operirten Pyämiefällen fand er 4 mal keine Sinus- oder Bulbusthrombose, wohl aber perisinuöse Herde, die in 2 Făllen allerdings so gering waren, dass ihre ursächliche Bedeutung $\mathbf{z}$ weifelhaf́t erscheint. In dem einen dieser Fälle ist wohl doch eine Bulbusphlebitis anzunehmen, während bei dem anderen Falle, einer schweren Scharlachotitis, die operativ gefundene Nekrose des knöchernen Sulcus sigmoideus - also eine Nekrose dicht am Sinus - die Lintstehnog der Pyămie mit Metastasen genügend erklärt. Bezüglich der Diagnose der Osteophlebitis meint Jansen, dass die bekannte Gegenüberstellung der 0 s te ophlebitis durch Körner, die sich gewöhnlich an acute Eiterungen anschliesse und zur Bildung von 
Gelenk- und Muskelmetastasen fübre, und der Sinusphlebitis, die meist durch chronis che Eiterungen bedingt, zu Lungen metastasen führe, weder nach den Zusammenstellungen aus der Litteratur noch nach seinen eigenen Erfahrungen zu Recht bestehe: Handelte es sich doch in den erwähnten, aus der Litteratur zusammengestellten 60 Fälen in $25 \%$ um chronische Fälle, bestanden doch in $25 \%$ Lungenmetastasen. Bei 13 Fällen Jansens, von denen 10 das Bild der Osteophlebitís-Pyämie, 3 noch geringere Symptone darboten, wurde durch die Operation stet s Sinus-oder Bulbusthrombose festgestellt. Wenn also die Osteophlebitis-Pyämie äberbaupt vorkommt, so ist sie extrem selten und daher ohne practische Bedeutung. Das Auftreten von Gelenk- und Muskelmetastasen gewährleistet nach Jansen's Erfabrungen gerade das Bestehen einer Sinus- oder Bulbuserkrankung. Die Periphlebitis des Bulbus entzieht sich fast immer unserer Wahrnehmung. Die Entstehung der Bulbusthrombose wird durch Dehiscenzen am Paukenboden, hochgelegenen Bulbus etc. begünstigt.

Betreffs der Behandlung der otitischen Pyämie herrscht in dem Punkte Uebereinstimmung, dass der septis che Thrombus entfernt werden muss, während die Frage der Jugularisunterbindung noch strittig ist, ebenso wie die Frage der Bebandlung wandstăudiger Thrombosen. Leichte Pyämien können schon durch Freilegung des Sinut zur Heilung gelangen. Die Sinuseröffnung mit Jugularisunterbindung ergiebt nach der Litteraturzusammenstellung bessere Resultate, als die Sinuseröffnung allein. Das beweist die Viereck'sche Statistik: Unter 94 Fällen war in 40 unterbunden, in 54 nur am Sinus operirt worden. Von den 40 Fällen sind 6 gestorben. 34 geheilt, von den 54 sind 13 gestorben, 41 gebeilt. Von den 41 Geheilten hatten aber 16 noch lange pyämisches Fieber, 11 Metastasen. Zaufal veriangt immer die Unterbindung vor der Operation. Körner hält die Unterbindung jmmer für gerechtfertigt. v. Berg man $n$ stellt sie jetzt in seinem Lehrbuch als empfehlenswerth hin, nachdem er sich früher viel bestimmter dafür ausgesprochen. Hessler und Brieger wollen sie nicht in allen Fällen ausgeführt wissen. Brieg er hält die prophylaktische Unterbindung für unnütz, ja schädlich. Leutert will, dass sie erst nach der Sinuseröffnung und nur unter bestimmten Voraussetzungen ausgeführt werde. Ma cew en scheint aus seinen Veröffentlichungen ist darüber keine Klarheit zu gewinnen in seinen Fällen nur die Sinusoperation ausgeführt zu baben, jedoch kein principieller Gegner der Unterbindung zu sein. Nach J. ist in allen Fällen von Sepsis oder schwerer Pyämie mit schlechtem Allgemeinbefinden und Lungenmestasen die Jugularisunterbindung vor der Operation wünschenswerth. In allen anderen Fällen ist nach der Knocbeneröffnung der Sinus freizulegen, und wenn sich jauchige Flüssigkeit in ibm zeigt und die Annahme besteht, dass die Infection bis zum Bulbus heruntergeht, sofort zu unterbinden. Bei solidem Thrombus im Sinus ist die Unterbindung überflüssig. Ist der Sinus mit fiüssigem Blut gefüllt, also entweder gesund oder mit wandständiger Thrombose erfüllt, so kann man den Effect der Operation abwarten, ohne den Sinus zu incidiren. Ist wandständige Thrombose nachgewiesen, so ist entscheidend für das Vorgehen das Algemeinbefinden: bei schwerer Pyämie ist $\mathrm{zu}$ unterbinden. Bei wandstāndiger Thrombose ist die Unterbindung möglichst hoch oben - über der Vena facialis -.., in den anderen Fällen am besten recht tief, besonders wenn Jugularisphlebitis vermuthet oder gefunden wird, auszuführen.

Der Sinus soll nur dann incidirt werden, wenn sich septische Thrombose zeigt, niemals zu diag nostischen Zwecken. Hierfür genügt die Punction mit der Nadel. Das Verfahren zur Erkennung der Bulbusbetheiligung (Druck auf die Jugularis nach Eröffnung der Sinus und Beobachtung, ob dabei von unten Blut oder Eiter nachfliesst) verwirft J. und empfiehlt dann noch eher die ungefährlichere Jugularisunterbindung. Auch das Whitling'sche Verfahren hält J. für eine nicht ungefährliche Maassnahme. J.'s eigene Erfahrungen erstrecken sich auf 50 Fälle, von denen 30 nur am Sinus, 20 auch an der Jugularis operirt sind. Von 10 am Sinus operirten Fällen sind alle geheilt, von 12 mit Unterbindung Operirten sind 3 gestorben. Unter letzteren 3 Fällen war bei 1, unter den geheilten Fällen 
war bei 2 der Sinus nicht incidirt worden. Im Ganzen waren J.'s Resultate in den letzten Jahren günstigere:

bei Sinusoperation in 100 Proc. Heilung
bei Jugularisoperation $=75=$
bei allen Operationen $=86,4=$

Herr Brieger-Breslau (Correferat: "Ueber den gegenwärtigen Stand der Lehrevon der otogenen Pyămie.") Die otogene Pyâmie hat keine absolut einheitliche Genese. In der überwiegenden $\mathrm{Zahl}$ der Fälle ist sie durch thro mbophlebitis che Prozesse - in einem Hirnblutleiter oder jm Bulbus jugularis - bedingt. Es kommen aber auch Fälle zur Beobachtung mit dem a s ges prochenen Bilde der Pyämie, bei denen auch anatomis ch normales Verhalten in allen zum Ohre in Beziehnng stehenden Venen bezirken gefunden wird. Der Einwand, dass in solchen Fällen der primäre thrombophlebitische Prozess schon zur Heilung gelangt sei, trifft allgemein nicht zu und hat am allerwenigsten für solche Fälle Bedentung, in denen so frische Metastasen nachgewiesen werden, dass man an der Stelle, die das Material für die Embolie geliefert haben soll, zu mindesten noch Spuren der Thrombophlebitis treffen müsste. $B$. berichtet über $\mathrm{z} w$ ei derartige von ihm erhobene Befunde von Pyämie obne den Befnnd der Sinusthrombose - Allgemeininfectionen, welche klinisch unter dem Bilde der Pyämie verlaufen und mit Metastasenbildung einhergehen, kommen vom $\mathrm{Ohr}$ aus direct ohne die Vermittlung von Sinusthrombose durch rein bacterielle Embolien zu Stande. Die Annahme, dass Osteophlebitiden in den Venen des Warzenfortsatzes das Substrat für die an acute Mittelohreiterungen sich auschliessenden, von Körner als Osteophlebitis-Pyämie charakterisirten Formen der Pyämie abgeben, ist unbewiesen. Die Sonderstellung dieser Fälle ist nicht a ufecht $z$ u erhalten; auch Sinusthrombosen mit vollständigem Verschlusse des Blutleiters nach acuten Eiterungen zeigen den gleichen Verlauf. insbesondere die gleiche Localisation der Metastasen. - Ein grosser Theil der Fälle, in denen normales Verhalten des Sinus bei Pyämie klinisch eruirt wird, ist durch den Nachweis des Vorkommens wandständig bleibender Thromben erklärt. Wandständige Thromben kommen, ohne das Bild der Pyämie hervorzurufen, im Sinus transversus, von benachbarten Entzündungsherden - bei Hirnabscessen, Meningitis, cariösen Processen der Pyramide - ausgehend vor. Die obturirende Thrombose des Sinus transversus, die sich noch im Stadium des Zerfalls durch das Fortbestehen eines Abschlusses am peripheren wie centralen Ende als solche oft characterisiert, ist entweder - gewöhnlich - durch das Wachsthum wandständiger Thromben oder durch die Fortsetzung einer Thrombose von in den Sinus mündenden Venen in diesen hinein bedingt. - Bei der Entstehung der otogenen Pyämie sind am häufigsten Streptococcen aber nicht ausschliesslich - wirksam. Auch Staphylococcen, Pneumococ. cen, Bacillus Friedländer sind als Erreger der Sinusthrombose nachgewiesen. Die Virulenz der Erreger ist sehr variabel und nicht immer des Schwere des Krankheitsbildes correspondirend. Die Mikroorganismen sind innerhalb der Thrombusmassen nicht gleichmässig vertheilt. Je nach Menge und Virulenz der in den einzelnen Abschnitten verbreiteten Erreger kommt es bei embolischer Aussaat nur zu einfacher Infarcierung oder zur Bildung metastatischer Abscesse. Einmaliges Ueberstehen einer Pyämie-Attake schützt auch auf kurze Zeit hin nicht gegen Recidive der Pyämie, welche sich bei unvollkommener oder ganz ausbleibender Heilung des Primärherdes einstellen können. - Die Diagnose der otogenen Pyämie kann nicht ohne Weiteres auf den Nachweis graduell fixierter Temperaturen aufgebaut werden. Auch bei uncomplicirten Eiterungen tinden sich gelegentlich selbst höhere Fieberbewegungen auf Tage hinaus. Nurdie charakteristische Curve ist einwandsfrei beweisend. Sie kann aber bei otogener Pyämie erheblich verändert sein: das Fieber kann bei Sinusthrombose vollständig fehlen. Verlauf in den metastatischen Herden und dem primären Prozess im Sinus correspondiren einander nicht immer. $Z$ wis chen den einzelnen Metastasen konnenmonatelange Intervalle liegen. - Aus der Inspection des freigelegten Sinus ergeben sich sowohl bei Betrachtung der äusseren Wand, als nach der Incision auch der medialen Wand wichtige Anhaltspunkte. Das Verhalten der pulsatorischen 
Bewegungen am Sinus, die Herstellung der Blutleere in dem zu explorirenden Sinusabschnitte finden ausführlichere Erörterung. - Die operative Behandlung findet ihre Contraindicationen nur in denjenigen allgemeinen Momenten, die als die Prognose jedes Eingriffs überhaupt ungünstig gestaltend gelten (Diabetes). Auch bei dusgesprochener Meningitis, beim Vorhandensein metastatischer Lungenabscesse, bei Fällen mit schwer toxischem Verlaufe ist operative Heilung beobachtet. Als Endziel der operativen Behandlung ist der Abschluss des Sinus in beiden Richtungen anzustreben. Deswegen ist die principielle Ausräumug auch der soliden Thrombusmassen an den Liden des Thrombus zu widerraten. Der zur Heilung wandständiger Thrombosen eingeschlagene Weg: aus der wandstäudigen, an sich direct nicht angreifbaren Thrombose durch Unterbindung der Jugularis eine obturirende zu machen und dann den Sinus direct anzugreifen, hat seine ernsten Gefahren. Bei der Eröffnung des nicht vollständig verstopften Sinus besteht die Gefahr der Luftaspiration unter gewissen äusseren, den Druck im Schädelinneren beeinflussenden Bedingungen. Für den Eintritt der Luft in die Venenbahn stellt die Jugularis den einzigen Weg dar; ihre Compression oder Unteroindung schützt sicher gegen diese Gefahr. Zur Verhütung der Verbreitung mobil gewordener Thrombusabschnitte ist die Jugularisunterbindung theils entbehrlich, weil in dem durch die Sinusthrombose aus der Circulation ausgeschalteten Abschnitt der Jugularis eine feste Verklebung ihrer Wände oft eintritt, theils schädlich, weil damit die Thrombose a uf b is dahin freigebliebene Strecken der Bahn sich ausdehnen kann. Die Ligatur giebt einen absolut sicheren Schutz gegen die Aus breitung der Phlebitis in der Continuität der Vene. Die Gefahren der Ligatur vermindern sich erheblich, wo man der Unterbindung die Spaltung des ligirten Abschnittes folgen lassen kann.

Discussion: Herr Körner-Rostock: Das Krankheitsbild der Osteophlebitis-Pyämie ist thatsächlich seltener, als $K$. früher annahm. Die Möglichkeit der Entstehung von pyämischem Fieber mit peripheren Metastasen durch Erkrankung des Knochens ohne Sinusbetheiligung ist aber doch vorhanden. Es müssen genaue anatomische Untersuchungen und klinische Beobachtungen in Zukunft erst die Frage der Osteophlebitis-Pyämie entscheiden. Ganz sicher wird man in absehbarer Zeit zu einer Klarbeit über diese Frage gelangen.

Herr L eutert-Königsberg: Die praktische Bedeutung der OsteophlebitisPyämic kommt nicht mehr in Frage, höchstens die Möglichkcit ihres Vorkommens. Allen otogenen Pyämieen liege Sinus-oder Bulbusthrombose zu Grunde. Bei negativem Sinusbefund obducirter Falle sei die mikroskopische Sinuswanduntersuchung nöthig. Wenn nach Ablauf des Initialstadiums uber 3 Tage eil Fieber von über $39^{\circ}$ anbalte, so sei Sinusthrombose wabrscheinlich. Dieses Schema müsse man den weniger erfahrenen Collegen an die Hand geben. Die nach Jansen diagnostische Schwierigkeiten bereitenden Fälle mit continuirlichem Fieber sind gerade die gefährlicnen. Eine nicnt inificirte l'hrombosenform könne er nicht anerkennen. Nach den Untersuchungen von Evers und Schimmelbusch entstehen Thrombosen nicht durch Verletzung, sondern durch chemische Blutinfection. Im Gegensatz zu Jansen meint er, dass bei Pyämie stets und zwar möglichst bald die völlig un gefäbrliche sinusincision vorzanehmen sei. Geringer Knochenbefund sei kein Grund, eine Sinusphlebitis auszuschliessen. Die Bulbusthrombose geht wohl nur selten von der Paukenhöhle aus. Nach L.'s jotzigem verauderten Standpunkt sei die Unterbindung immer und zwar als erster Operationsact auszuführen. Die Gefahr, dass nach Unterbindung die Thrombose auf den Sinus petrosus übergehen könne, sei theoretisch construirt, durch keinen Fall bisher praktisch nachgewiesen. Wenn anzunehmen ist, dass die Thrombose bis in die. Jugularis heruntergeht, so sei mögiichst weit unten zu unterbinden, sonst immer oben. Die- Probepunction des Sinus köune er nicht emptehlen. Wenn nach der Incision sehr starke Blutung auftrete, brauche man nur 1 bis 2 Tage zu warten. Das Whitling'sche Vertahren ist nicht immer anwendbar. Erst vor 2 Tagen sei ihm bei seiner Anwendung der Sinus eingerissen.

Archir f. Ohrenheilkunde, LII. Bd. 
Herr Panse-Dresden: P. nimmt das Whitling'sche Verfahren in Schutz. Die Osteophlebitis-Pyämie kann durch Bulbusthrombose vorgetäuscht nerden. Bei der Entstehung der letzteren kann der hobe Druck in der Pauke keine Rolle spielen, da Bulbustbrombose auch in Fällen mit grosser Trommelfellperforation beobachtet worden sei.

Herr Habermann-Graz: H. erwähnt einen letal verlaufenen Fall seiner Beobachtung, in dem trotz Bestehens einer grossen Perforation des Trommelfells der Bulbus jugularis rom Boden der Pauke aus inficirt wurde. Der Thrombus im Bulbus jugularis wurde erst bei der Cntersuchung des herausgenommenen Schläfenbeines aufgefunden.

Herr Eren fried-Berlin: E. berichtet über einen 20 jährigen Patienten mit langjähriger Atticuseiterung und Knöchelchencaries, bei dem nach Atticussondirung schwerer Schwindel auftrat. Am nächsten Tage Fieber mit Schütteifrost. Ueberführung des Patienten in die Körte'sche Klinik. Dort wird ein Ellbogenabscess eröffnet. Schwirdel sistirt am 11. Tage. Nach 4 Wochen Heilung obne Operation.

Herr Denker-Hagen: Ob die Osteophlebitis-Pyämie möglich ist, muss durch eine grössere Zahl yon Sectionen entschieden werden. Das wird aber bei der Seltenheit dieser Fälle noch lange dauern. D. berichtet einen Fall von Influenza-Otitis mit Gelenkmetastasen und völliger Einscbmelzung des kranken Warzenfortsatzes. Der Umstand, dass das Fieber nach operativer Beseitigung der Metastasen am Oberarm und Daumengelesk sistirte, macht es wabrscheinlicb, dass hier eine Osteophlebitis-Pyămie ror]ag.

Herr Schwabach-Berlin: Dass pyämische Erscheinungen durch Bacteriämie, wie Herr Brieger erwähnt, bedingt sein können, belegt Sch. durch folgenden Fall : 22jăhriges Mädchen mit doppelseitiger chronjscher Eiterung. Pyämie. Jugularis empfindlich. Radicaloperation. Sinus incidirt; enthält flüssiges Blut. Nach 2 Tagen Exitus. Bei der Obduction (Benda) in keincm Sinus eine Spur von Thrombose zu finden. Metastasen nur in den Nieren, die fast ganz aus Streptococcen bcstebende Infarcte in den Papillen aufwiesen.

Herr Hofmann-Dresden: In meinem von Herrn Jansen erwäbnten Falle war die Diagnose auf Sinusthrombose gestellt, die Unterbindung aber wegen schlechter Narkose erst später ausgeführt wordev. In meinem von Herrn Leutert erwähnten Falle schloss sich an die Verletzung des Sinus ausgedehnte Thrombose des Sivus transverus und cavernosus an. Die Verletzung muss ihr Entste hen veranlasst haben.

Herr Alt - Wien: A. stirmt Herrn Jansen darin bei, dass man bei der Jugularisunterbindung radical vorgehen, das ligiıte Venenstück reseciren solle. A. erwähnt 2 Fälle von wandständiger Thrcmbose, bei denen er die Unterbindung vornalm. Bei dem einen trat perisincöser Abscess und Periphlebitis mit Balsphlegmone, bei dem zweiten sccundärer eitriger Zerfall im unterbundenen Jugularisstumpfe auf.

Herr Scheibe-München: Die besonders bei acuten Fällen auftreterde is olirte Bulbusthrombose scheint sich besonders gern an Influenzaeiterungen anzuschliessen. Sch. erwähnt eiren Fall von Influenza mit grosser Perforation, ohne Eiterung, in $d \mathrm{tm}$ es zu Bulbustbrombose kam. Hier konvite also Druck von Eiter als ätiologisches Momeut nicht im Spiele sein. Der Influenzabacillas befalle gern Biutgefässe und rufe nicht selten Nekrosen hervor. In der Literatur finden sich eine Anzahl Fälle von isolirter Bulbusthrombose bei Influevza, z. B. '3 Fälle aus der Hallenser Klinik.

Die weitere Discussion wird auf morgen verschoben. Schluss der Sitzung $1 / 21$ Thr:

\section{Sitzung am 24. Mai, Naehmittags 1/23 Uhr.}

11. Herr Alt-Wien: Ein Beitrag za den musikaliscben Hörstörungen: Nachdem $A$. über einen sehr interessanten Fall von nach seiner Ansicht perjpherentstandener completer Tontaubbeit berichtet hat, schildert er seine exper:mentellen Ul tersuchungen behufs Ei mittlug g der Frage, ob und inwiefern durch Schallleitungshindernisse Störungen des musikalischen Gehörs auftreten bönen. A. exzeugte an normalen Ohren ein Eirwärts- 
drängen des Trommelfells und der Ossicula, indem er mit 2 mit Schlanch und Ballon versebenen Siegleschen Trichtern die Gehörgänge hermetisch verschloss und dann einen oder beide Ballous so stark comprimiren liess, dass der durch Aufsetzen einer Stimmgabel auf den Scheitel im Ohr erzeugte Ton nur abgeschwächt, nicht aufgehoben wurde. Da sich bierbei belastete und unbelastete Stimmgabeln wegen zu kurzer Schwingungszeit als unverwendbar erwiesen, benutzte A. König'sche Stimmgabeln mit elektromotorischem Antrieb. Zur Tonübertragung wurde Knochenleitung verwendet (Proc. mastoid. bzw. Stirn bei doppelseitiger Prüfung). Schon durch den leisesten Druck des Ballons wurde der Grundton bei doppelseitiger Compression zum Verschwinden gebracht; bei weiterer Drucksteigerung verschwanden auch die Obertöne. Bei einer zweiten Versuchsanordnung, bei der 2 Stimmgabeln (eine c-Gabel mit 32 und eine mit 250 Schwingungen) eingespannt wurden, verschwand der Ton der tieferen Gabel schon bei mässigem Fingerdruck in die Gehörgänge, während der Ton der höheren Gabel abgeschwächt wurde. Viel eclatanter war dieses Phänomen bei Anwendung des Compressionsballons an den Siegle'schen Trichtern. Weniger sinnfälig fielen diese Versuche bei Anwendung von Pfeifen aus. Es trat hier eine $\mathbf{A b}$ schwächung beider Töne, aber eine ungleich grössere für die tiefen Töne ein. Die Versucbe zeigen, dass schon ein mässiges Einwärtsdrängen des Trommelfells und der Ossicula dje Tonempfindung wesentlich beeinflussen kann. (vgl. den Gellé'schen Versuch. -.. d. Ref.). Es fragt sich nun, ob die Abschwächung oder Aufhebung der Perception für tiefe Töne auf der straffen Anspannung der Ossicula oder auf Steigerung des Labyrinthdrucks beruht. Zweifellos stellt letztere die Ursache dar. Es handelt sich um eine durch Compression bewirkte Belastung der für die tiefen Töne bestimmten Fasern der Membrana basilaris. Bezüglich der Function der Ossicula nebst ibren Muskeln schliesst sich A. der bekannten Zimmermann'schen Auffassung an. Bei Mittelohrprocessen wird die druckregulatorische Wirkung der Ossicula auf die Labyrinthflüssigkeit durch die straffe Spannung der Knöchel gestört oder durch Belastung des runden Fensters durch Exsudat bei Mittelohrkatarrhen, die ja am häufigsten Diplacusis erzeugen. $\mathrm{Zu}$ berücksichtigen ist auch, dass nach Kiesselbach durch die Contractionen des bei gespannter Kette durch mässige Schallreize zu reflectorischer Innervation gelangenden Musc. tensor tympani bei tiefen Stimmgabeln ein Schwächerwerden und Höherwerden des Tones, bei mittleren nur ein Schwächerwerden und bei höheren Tönen keine Veränderung erzengt wird. Durch den Wegfall der tiefen Töne können die Obertöne so hervortreten, dass sie vom kranken Ohre allein gehört werden und Patient einen böheren Ton zu hören glaubt. Bei den meisten Djplacusisfällen war ein Höherhören zu beobachten. Solche Diplacusisfälle, die nur bei gesonderter Untersuchung beider Ohren nachweisbar sind, manifestiren sich gewöhnlich nicht als Falschhören. Zum Falschhören kommt es, wenn 1. das andere Ohr für den Höract von früher her wenig geeignet ist, 2 . bei neurasthenischen, sensiblen Musikern die Störung der Klangform Doppelthören erzengt, 3. mit der Mittelohraffection eine Labyrintherkrankung combinirt ist. Die meisten Diplacusisfälle betreffen acute oder chronische Mittelohrkatarrbe oder Residuen derselben. Die Aetiologie des Falschbörens bei diesen Prozessen glaubt A. durch seine Ausführungen erklärt zu haben. Auch die beiden von Grunert und Dennert beobachteten Diplacusisfälle bei fehlendem 'Trommelfelle lassen sich in gleicher Weise erklären. Eine grosse Rolle für das Verständniss dieser Erscheinungen spielen auch Hysterie, Neurasthenie, Ermüdung des* Ohres und geistige Abspannung. Ein feinhöriger Musiker kann, zumal wenn er nervös ist, durch Sausen oder Autophonie eine wesentliche Beeinträchtigung seines musikalischen Gehörs erleiden. Ueber eine zweite Versuchsreihe zur Exklärung der Diplacusis echotica und äber Falschhören durch Labyrintherkraukung wird $\mathrm{A}$. an anderer Stelle berichten.

Discussion:

Herr Dennert-Berlin: Nicht selten beobachtet man bei Patienten die Unfähigkeit, die Differenz verschiedener Töne wahrzunehmen. Ein Patient z. B. konnte nur Töne unterscheiden, die um 2 Octaven different waren; 
aufeinanderfolgende Töne erweckten bei ihm keinen dysharmonischen Eindruck. Bei starken Mittelohrkatarrben und -Entzündungen wird Musik von Patienten ofters als eine Kette unangenehmer Dysharmonieen empfunden.

Herr A lt-Wien: Der von A. erwăbnte Patient börte nur den Rhythmus der Musik, die ihm im Uebrigen nur als ein unangenehmes Geräusch imponirte. Cerebrale Störungen, Hysterie waren hier anszuschliessen. Es bestand demnach complete periphere Tontaubheit. Es haben hier beide Ohren falsch gehört. Das linke $\mathrm{Ohr}$ hörte jeden Ton um $1 / 2$ Ton höher, das rechte um 2 Töne höher. Ein solcher Fall ist in der Litteratur noch nicht beschrieben. Herr B erthold-Köngsberg: B. berichtet über einen Musiker mit perforativer Otitis media, bei dem die viel seltenere Diplacusis monauralis im Bereich von $\mathrm{c}_{1}-\mathrm{ca}$ auftrat.

Herr $P$ a n $\mathrm{e}$-Dresden: $P$, bält es nicht fur erwiesen, dass im Falle des Herrn Alt die Tontaubheit nicht central bedingt war. Denn penn Patient Musikpartituren mit Genuss lesen konnte, so beweist das zwar, dass die corticale Empfindung intact war, schliesst aber subcorticale Störungen keineswegs aus.

Herr E hrenfried - Berlin: Wenn die Tontaubheit in dem von Herrn Alt angeführten Falle darauf berubt, dass rechts um 2 Töne, links um $1 / 2$ Ton höher gehört wird, so muss bei Ausschaltung cines Ohres die Tontaubheit verschwinden.

Herr Alt-Wien: Bei Anwendung von Musik, von starken Klăngen, wie sie fưr Schwerhörige bei der Prüfung nöthig sind, kann man nicht ein $\mathrm{Ohr}$ ausschalten. Ein Centrum für die musikalische Empfindung ist im mittleren Theil der zweiten linken Schläfenwindung angenommen worden. (Gegen die Richtigkeit dieser Annahme spricht ein Fall von erworbener Amnesie aus der Beobachtung von Mann-Breslau, bei dem autoptisch sich eine Herderkrankung im rechten Stirnhirn fand. D. Ref.)

III. Herr De utschländer-Breslau: Demonstration eines im Mai 1899 an Mittelohrentzündung erkrankten Pat, bei dem schliesslich durch Herrn Kü mmel die Radicaloperation ausgefübrt werden musste. Deutschländer entschloss sich als Vertreter des Herrn $K$ ü $\mathrm{mmel}$ bald darauf wegen entsprechender Erscheinungen das Labyrinth zu eröffnen, das sich indess intact erwies. Bei der erforderlichen nächsten Operation wurde unter Schonung des Facialis, der völlig wie im anatomischen Präparat freigelegt wurde, der ganze Warzenfortsatz bis zum Processus stylödes abgetragen. Es fand sich Eiter in der Tiefe. Entleerung eines perisinuösen Abscesses. Pat. fieberte beständig weiter und zeigte eine Schwellung in der Jugularis- und Nackengegend. Da ein Senkungsabscess in der Jugularisscheide vermuthet warde, weil durch Druck auf die Jugularisschwellung sich Eiter nach oben entleerte, wurde die Jugularis freigelegt. Dieselbe erwies sich indess als intact. Bei einem Druck auf die Occipitalgegend zeigte es sich, dass sich Eiter, der sich nach dem Atlantooccipitalgelenk einen Weg gebahat hatte, nach der Wundhöhle entleerte. Deutschländer verlängerte uun den öberen und unteren Operationsschnitt, verband beide, löste den Sternocleidomastoïdeus los, bahnte sicb einen Weg zur Fossa pterygomaxillaris, wobei der Facialis geopfert werden musste, und drang dann stumpf mit dem Finger gegen las erwähnte Gelenk vor, aus dem aller Eiter entleert wurde. Sofort nach dieser Operation sistirte das Fieber and war der Krankheitsprocess wie abgeschnitten.

IV. Herr W. Stern (Doc., Dr. phil. als Gast) Breslan: Stern demonstrirt seinen Tonvariator, eine continuirliche Flaschen-Tonreihe, bestehend aus 4 Flaschen, die das Tongebiet der kleinen, der ein- und zweigestrichenen Octave umfassen. Der Ton wird erzengt durch Anblasen der in der Flasche befindlichen Luftsăule mittels Blasebalgs. Die Tonveränderung wird dadurch erzengt, dass aus einem mit Wasser gefüllten Cyliader durch Vorwärtsbezw. Rückwärtsbewegung eines Kolbens Wasser in die Flasche hineingeführt bezw. aus itr in den Cylinder zurückgeleitet wird, d. h. also durch Verkleinerung bezw. Vergrösserung der in Schwingung versetzten Luf́tsäule in der Flasche. Die Geschwindigkeit der Tonveränderung ist eine gleichmässige, der Geschwindigkeit der Kolbenverschiebung proportionale. Die Gleichmässigkeit der Veränderung wird durch die mit den Flaschen verbundenen Regulirtrichter erzielt, durch deren Eiuschaltung das Wasser mit abnehmender Geschwindigkeit in die 
Flaschen aufsteigt. Diese genau in ibrer Form berechneten Trichter müssen deshalb eingeschaltet werden, weil in den oberen Regionen der Flasche $-d . h$. bei grösserer Füllung derselben - der Ton sich schneller ändert, als in den unteren. Die Vorzüge des Apparates gegenüber den Stimmgabeln sind folgende: 1. Die erzeugbaren Töne bilden eine Wirklich e continuirliche Reibe, $d$. h. sie können ohne Unterbrechung hinter einander erzeugt werden, wodurch bei Hörprüfungen eine grosse Zeitersparnis gegenüber den anderen Verfahren bedingt wird. 2. Man kann jeden Ton beliebig lange und mit constanter Intensität ertönen lassen. 3. Man beherscht mit dem Apparat die feinsten Tondifferenzen, Unterschiede von einer oder wenigen Schwingungen, ja ev. von Bruchtheilen einer Schwingung. Der Nachtheil des Apparates liegt in seinem beschränkten Tonumfang. Gerade die für das Sprachverständniss erforderlichen Töne (b $b_{1}-g_{2}$ nach Bezold) entbält aber der Apparat. (Letzter Punkt wichtig fừ die Verwendung des Apparntes bei Taubstammenuntersuchungen. d. Ref.)

\section{Discussion:}

Herr Berthold-Königsberg: Bei belasteten Stimmgabeln sind die Töne, wenn die Gewichte an den Enden der Gabeln sich befinden, lauter, wenn die Gewichte heruntergeschraubt werden, schwächer. Also bei diesen Stimmgabeln tritt eine Veränderung der Intensität des Tones mit der wechselnden Höhe desselben - bei ein und derselben Gabel - ein.

Herr Denker-Hagen: Der Apparat wird erst dann eine practische Wichtigkeit für Hörprüfungen erlangen können, wenn die untere und obere Tongrenze in sein Bereich fallen. Die Töne scheinen Denker nicht ganz obertonfrei zu sein. Herr Denker fragt an, ob sie auf Obertöne geprüft sind.

Herr Stern-Breslau: St. verneint die Frage, betont aber, dass schon Helmholtz und Stumpf diese Flaschentöne für den Stimmgabeltönen fast gleichwerthige, nahezu obertonfreie Töne erklärt haben.

Herr Panse-Dresden: Mit dem Stern'schen Apparat wird man nachweisen können, ob hohe oder tiefe Töne für das menschliche Obr stärker wahrnehmbar sind.

Herr Dennert-Magdeburg: Mit Sirenen kann man wohl dasselbe erreichen wie mit dem interessanten $S$ tern'schen Apparate.

Herr Stern: Die durch Birenen erzeugten Töne sind sehr obertonreich, also mit den Flaschentönen nicht in Parallele za stellen. Herr Stern be. tont noch als einen weiteren Vorzug seines Apparates, dass, während der Uebergang ron den höchsten Stimmgabeltönen zu den tiefsten Pfeifentönen der Bezold-Edelmann'schen continuirlichen Tonreihe ein bezüglich der Ton-Intensität sebr abrupter sei, bei seinem Apparat die Tonübergänge viel bomogener seien.

V. Die Herren Peters und Hinsberg-Breslau: Demonstration von Plattenmodellen über die Entwicklung der Nase bei Wirbelthieren und Menschen.

VI. Herr Berthold-Königsberg: Weitere Mittheilungen zur intranasalen Vaporisation. Berthold demonstrirt einen modificirten Pin cuss'schen Apparat mit Ansatzstücken für die Nase, Kieferhöhle u. s. W., durch dessen Anwendung eine fibrinöse Entzündung der betreffenden Schleimhaut mit darauffolgender Neubildung normal functionirender Schleimbaut erzeugt werde.

VII. Herr S cheib - München : S c l e ib e demonstrirt ein vorn geknöpftes Messer mit kurzer, schwach gebogener Schneide zur Abtragung der Lateralstränge des Pharynx. Während ein Assistent die Zunge niederdrückt, wird der Strang mit der linken Hand mittels eines Zängelchens nach vorn gezogen, mit der rechten Hand von oben nach unten abgeschnitten.

VIII. Herr Kayser-Breslau: 1) Demonstration eines von einem 12 jährigen Knaben stammenden $N$ asensteines vom Pflaumengrösse. 2) Demonstration eines Pat. mit einem tuberculösen Ulcus im äusseren Gehörgang. Dasselbe hat seinen Sitz an der hinteren Wand und $z$ war im hintersten Theil des knorpligen Abschnittes, zeigt wallartige Ränder und ist mit einem gelblichen, eitrigen Secret bedeckt, in dem - schon im ersten Präparate - sich spärliche Tuberkelbacillen fanden.

IX. Herr Brieger-Breslau: 1) Demonstration eines Falles pon primärer Schläfenbeintuberculose bei einem 8 jährigen Mädchen, bei dem 
es zum Durchbruch nach der hinteren Schädelgrube und ausgedehnter Pachymeningitis tuberculosa gelzommen war. Der Fall ist operativ völlig geheilt worden, Die Pat, zeigt normales Trommelfell. 2) Demonstration des Scbläfenbeines ron einem 7 jäbrigen Mädchen, das mit schwerer Otitis media purulenta, vielen Ohr-Polypen und Taubheit in B.'s Behandlung kam. Die mikroskopische Untersuchung ergab Tuberculose der Paukenschleimhaut. Im Labyrinth zeigten sich bindegewebig ausgeheilte Entzündungsprozesse. Dass die Paukentuberculose secundär die Labyrinthveränderungen ausgelöst habe, ist möglich, aber unwabrscheinlich, weil für Tnberculose characteristische Erscheinungen im Labyrinth fehlen. Wahrscheinlich stellte sich erst acute Entzündung in der Pauke, dann im Labyrinth ein, und die Tuberkelbacillen wanderten erst secundär in das eiternde Mittelohr hinein. opticon.

$\mathrm{X}$. Demonstrationen mikroskopischer Präparate am Ski-

a) Herr Görke-Breslau: G. demonstrirt 1) mikroskopische Präparate 2weier Tälle von Fibrosarcom des Acusticus. In dem ersten dieser Fälle fand sich partielle Atrophie des intralabyrinthären Nerven und der Ganglienzellen, im zweiten volistăndige Atrophie derselben und Schwund des Cortischen Organes.

2) Schnitte durch Ohrpolypen. Die histologischen Befunde dieser Fälle sind ausführlich in diesem Archiv $\mathrm{Bd}$. 52 veröffentlicht.

Discussion zu 2): Herr $\mathbb{K} u ̈ m m e l-B r e s l a u: ~ K$. hält die als solche angesprochenen Gebilde nicht für Drüsen. Herr Habermann-Graz fragt an, $\mathrm{ob}$ in dem ersten Falle es sich um allgemeine Lymphomatose handelte. Herr Görke: Sobald die Schleimbant Epitheleinsenkungen in die Tiefe zeigt, die secernierende Zellen enthalten, die also anatomisch und physiologisch den Character von Drüsen aufweisen, sind dieselben anch als Drüsen zu bezeichnen. In dem fraglichen Falle bestand keine allgemeine Lymphomatose. Die Polypen stammen dort von der Paukenschleimhaut oberhalb des Trommelfells her, wo auch normaler Weise Follikel gefunden worden sind.

3) Schnitte durch das ausgeheilte, bindegewebige Processe aufweisende Labyrinth des zweiten von Herrn Brieger soeben besprochenen Falles.

b) Herr K ü m mel-Breslau demonstrirt ein Präparat von U ebergang der Eiterung aus der Pauke nach dem Sinus caroticus (Phlebitis des Sinus caroticus).

\section{Schluss der Sitzung um 5 Uhr Nachmittags.}

\section{Zweiter Sitzungstag, Sonnabend den 25. Mai.}

Morgens 8 Uhr folgten die Versammlungstheilnehmer einer Einladung der städtischen Verwaltung zur Besichtigung der Abtheilung für Ohren-, Nasen- und Halskranke am Allerheiligen-Hospital. Die Abtheilung - bekanntlich eine Stiftung Jacobys, an den eine Gedenktafel im Vorraum der Poliklinik erinnert - bat im letzten Jahre eine weitere Vermehrung ihrer Bettenzahl erfahren und kann jetzt 50 Kranke aufnehmen. Im Erdgeschoss befinden sich die Räume der Poliklinik - Wartezimmer, Behandlungszimmer, Dunkelräume -, ausserdem die Laboratorien, das Operationszimmer, Inhalatorium und Nebenräume. Im ersten Stockwerk befinden sich die Krankensäle, Einzelzimmer, Baderaum mit Schwitzbett, Zimmer für die Schwestern und andere Nebenräume. Die Einrichtungen der Abtheilung fanden den vollen Beifall der Besucher. Herr Dr. Brieg er demonstrirte ausser verschiedenen Apparaten und Instrumenten insbesondere einen von $G$. Härtel hergestellten Apparat für Heissluftbehandlung, sowie mehrere interessante Krankheitsfälle - Hirnabscess, Sinusthrombosen, Taubheit bei Leukämie, Tuberculose der Nase etc.

Um $9^{1 / 2}$ Uhr wurde die wissenschaftliche Sitzung mit der Fortsetzung der Discussion über das Referat der Herren Jansen und Brieger eröfnet. Disenssion:

Ilerr v. Wild-Frankfurt a/H.: Man sollte von otogener Allgemein. infection sprechen, in die man die relativ seltene Pyämie, Septicämie, Bacte- 
riämie und Toxicämie einzurechnen hätte. Wenn Herr Leutert Sinusthrombose und Pyämie identificiren wolle, so erinnere er daran, dass Allgemeininfectionen auch ohne primären Eiterherd oder Thrombose entstehen können. Beim Menschen ist z. B. Septicămie nach Angina beobachtet worden. Durch $\mathrm{K}_{0} \mathrm{ch}$ ist nachgewiesen worden, dass obne Erzeugung von Eiterberden durch Injection von Bacterien wahre Pyämie entstehen kanu. Wir dürfen uns für die Otologie keine eigene Pathologie construiren. Es ist nicht erwiesen, dass kleine wandständige Thromben der Ausgangspunkt einer Pyämie sein können; dagegen kann die Aligemeininfection sicher direct übertragen werden. Bei frübzeitig in Behandlung kommenden Allgemeininfectionen solle man sich also damit begnügen, den primären Herd auszuräumen, was keine Gefahr bringe, und nur wenn die Infection weitergehe, den Sinus anfsuchen und event. operiren.

Herr Preysing-Leipzig: Leutert's Behauptung, dass die Bulbusthrombose selten sei, ist falsch. Für die Forderung Le utert's, dass die Unterbindung der Sinusoperation voranzugehen babe, ist der angeführte Fall von Vereiterung im Venenstumpf nicht beweiskräftig; denn bei jeder Unterbindung eines thrombosirten Gefässstückes komme es zur Vereiterung des Stumpfes. Es ist ein ubertriebener Schematismus, zu sagen - wie es Leutert thut - dass eine Sinusthrombose vorliege, wenn lănger als 3 Tage das Fieber einen willkürlich angenommenen Grad überschreitet $\left(39^{\circ}\right)$. Das Fieber feblt ja bei Sinusthrombose öfters ganz. Solange nicht klare Symptome bestehen, soll man bei Behandlung der Sinusthrombose nur langsam, zögernd vorgehen. P. unterscheidet 3 Formen von Sinusthrombose: 1) Die organisirte Thrombose. In diesen meist fieberlosen Făllen ist die Unterbindung überfüssig; angezeigt höchstens, wenn nachträglich Fieber auftritt. 2) Formen, bei deneı der beiderseits solide, obturirende Thrombus nur in der Mitte eitrig zerfallen ist. $\mathrm{Ob}$ man hier unterbinden soll oder nicht, darüber werden immer $\mathrm{zwei}$ Meinungen herrschen. Bei fehlendem Fieber kann man nach $\mathrm{P}$. in diesen Fâllen mit der Unterbindung warten. 3) Formen, bei denen schon vor der Knochenoperation eine bis zur Jugularis reichende Sinusthrombose zu erkennen ist. Hier muss die Unterbindung den ersten Operationsact darstellen. In seinen letzten Fällen hat $P$. in der Mitte des Halses, wo die Carotis communis unterbunden wird, unterbunden. P. tastet sich hierbei bis zur Vena facialis herauf und unterbindet auch sie.

Herr Walliczek-Breslau:-W. führt einen von ihm beobachteten Fall von Bulbusthrombose bei Influenza-Otitis an, der die Behauptung Jansens betreffs der diagnostischen Schwierigkeiten jener Affection bestätigt. Es bestand in W.'s Falle kein Fieber, keine Schüttelfröste. Amnestische und motorische Aphasie. Lähmung des rechten Armes und Beines. Stauungspapille. Es fand sich operativ ein kleiner Hirnabscess in der mittleren Schädelgrube and nach hinten unten von ihm ein zweiter, grösserer Abscess. Patient starb in den nächsten Tagen. Die Section ergab ausser den beiden Hirnabscessen eine isolirte Bulbusthrombose. Der Thrombus war nach dem Sinus zu locker, der Sinus eiterfrei. Nach unten war der Thrombus solid outurirend. In der Jugularis tlussiges Blut.

Herr Deatschländer-Breslau: D. berichtet über einen Fall von Septicopyämie mit Schüttelfrösten und Ellbogengelenkmetastase, bei dem erst die äusserlich normal erscheinende Jugularis unterbunden, dann der sklerosirte Warzenfortsatz eröffnet wurde. Im Antrum nur ein kleiner Granulationsknopf und etwas Eiter. Am Sinus alles normal. Wunde heilte schnell. Nach der Operation noch ein Schuttelfrost. dann Erkrankung mit einem Schlage za Ende.

Herr L eutert-Königsberg: bemerkt Herrn Pre ysing gegenüber, dass er selbst gerade auf die $\mathrm{Hă} u \mathrm{figkeit}$ der Bulbusthrombose hingewiesen habe.

Herr Jansen-Berlin (Schlusswort): J. freut sich, dass die Beseitigung der Gegensätze zum Theil eingetreten ist. Er habe sagen wollen, dass wir bei den Fälien mit continuirlichem Fieber, die nach unserer übereinstimmenden Auffassung gerade die schwereren sind, die Diagnose der Meningitis schwer auszuschliessen vermögen. Der Nachweis von Coccen oder Eiterkörperchen in dem durch die Lumbalpunction gewonnenen Liquor cerebro- 
spinalis beweist nicht das Bestehen einer unbeilbaren, diffusen Meningitis, denn J. sah Fälle mit derartigem Befund heilen. J. sträubt sich nicht gegen die Jugularisunterbindung in sicheren Fälien. Die Ungefährlichkeit der Sinusverletzung kann er nicht zugeben. Als Beleg führt J. einen eigenen Fall an ron operativer Sinusläsion mit Blutung und normalem Wundverlauf. Nach 3-4 Wochen Magenkatarrh, Erbrechen, Kopfschmerzen, Fieber. Die jetzt ausgeführte zweite Operation ergab eine eitrige Sinusthrombose. Der Fall endete letal. In diesen Fällen ist der durch die Läsion erzeugte Thrombus zuerst nicht infectiös, kann aber inficirt werden, weil die Wunde nicht ganz rein zu halten ist. Leutert's Forderung, auch bei solidem Thrombus immer $\mathrm{zu}$ unterbinden, gehe entschieden $\mathrm{zi}$ weit. Nur inficirte Herde seien aus dem Körper auszuschalten. Erstaunt war J. über die Ansicht Leatert's, dass die Bulbusthrombose, deren Häufigkeit - und zwar besonders bei acnten Făllen - er zugiebt, vom Sinus aus entstehen müsse. Eine Eiterung am Paukenboden, dem der Bulbus, oft nur durch eine papierdünne Knochenwand getrennt, anliege, könne doch sehr leicht auf den Bulbus übergehen. Die Sinusincision ist kein ungefährlicher Eingriff. Ungeübten können bei ibr Entgleisungen rorkommen: sie können statt des Sinus die Dura incidiren, bei zu weit hinten ausgefübrter Incision ins Cerebellum geraten etc. J. behălt die Probepunction des Sinus bei, die nach seiner Ueberzeugurig nicht zu diagnostischen Irrthämern Veranlassung geben kann. Auch J. hält wie Preysing die Unterbindung in der Höhe der Cartilago cricoidea für gut. In Fällen aber, wo allein oder vorwiegend Bulbusthrombose besteht, ist die weiter oben ausgefübrte Unterbindung rathsamer. Bezüglich der Unterbindung präcisirt J. schliesslich seinen Standpunkt nochmals folgendermaassen: Die Unterbindung bat den ersten Operationsact $\mathbf{z u}$ bilden bei allen Fällen zweifelloser Sinusphlebitis und bei allen schweren Pyämien. Sie ist erst nach der Eröffnung des Sinus vorzunehmen, wenn der gefundene Thrombus über den Bulbus nach unten reicht oder wenn nach Eröffnung des Sinus Fieber und Schüttelfröste nach 2-3 Tagen nicht sistiren; sie ist nach der Freilegung des Sinus auszufübren, wenn der Sinus gesund erscheint, keine Periphlebitis besteht und trotzdem wiederholte Schüttelfröste auftreten, ebenso bei wandständiger Thrombose unter den letztgenannten Bedingungen (Schüttelfröste). In allen übrigen Füllen ist zunächst nicht $z u$ unterbinden.

Herr Brieger-Breslau (Schlusswort): B. glaubt, dass Herr Leutert an seiner principiellen Gegnerschaft gegen die Pyämie ohne Sinustbrombose nicht gut festhalten kann. So gut wie von anderen Stellen im Körper, können auch vom Sinus Bacterien in den Kreislauf gelangen und Pyämien erzeugen. Wir wissen, dass Bacterien, in Venen hineingebracht, manchmal schon nach Minuten in die entlegensten Körperstellen verbracht werden. Auch bei völlig abgelaufener Entzündung in Pauke und Antrum kann ausgesprochene Sinusthrombose bestehen. Die Bedentung von Dehiscenzen am Paukenboden für die Entstehung der Bulbusthrombose kann B. nicht anerkennen; denn die hierbei in der Regel bestehenden bindegewebigen Verschlüsse bieten vielleicht einen besseren Schutz dar, als der dunne Knochen. Die klinische Diagnostik bedarf eines weiteren Ausbaues um so mehr, als von bacteriologischer Seite in diesen Fragen kaum etwas zu erwarten ist. Den Leutert'schen Schematismus hält B. für eine Gefahr, da die unnütze Sinuseröff́nung nicht ungefăhrlich ist. Wenn man Fälle sieht, bei denen die zur Unterbindung kommende Jugularis wegsam ist, dagegen nach 2 Tagen thrombosirt erscheint, und schon theilweisen Zerfall des Thrombus aufweist, so muss man zugeben, dass wohl die Ligatur plus In fectionsgelegenheit d. i. event. eine wandständige Thrombose, schliesslich zur obturirenden Thrombose führen kann. Die Verletzung des Sinus führtnichtzur Thrombose, wobl aber unbedingt die Einfürung eines Tampons in das Sinusinnere. Erzeugen doch alle Fremdkörper, in Venen eingeführt, daselbst Thrombose.

Es wird nun eine kurze geschäftliche Sitzung eingeschoben:

Zunächst wird ein Danktelegramm des Herzogs Johann Albrecht 
จ. Meklenburg-Schwerin verlesen. Der vom Ausschuss für die nächste Versammlung zum Vorsitzenden erwahlte Herr Siebenmann-Basel hat die Wahl telegraphisch dankend angenommen. Zu seinem Stellvertreter ist Herr Bürkner-Göttingen erwählt worden. An Stelle des Herrn Kessel-Jena, der telegraphisch mittheilte, dass er auf seinem Ausscheiden aus dem Ausschuss beharre, wird Herr Kretschmann-Magdeburg von der Versammlung in den Ausschuss gewählt. Sodann werden 3 Anträge des Herrn HartmannBerlin von der Versammlung angenommen. Der erste derselben bestimmt, dass für die inzwischen auf über $4000 \mathrm{M}$. angewacbsene v. Tröltsch-Stiftung weitere 1000 M. aus dem Vermögen der Gesellschaft beigesteuert werden. Dem zweiten zufolge wird der Ausschuss mit einem Statutenentwurf behufs Eintragung der Gesellschaft in das Vereinsregister beauftragt. Dem dritten Antrag gemäss wird die Gründung einer Vereinsbibliothek beschlossen. Als Ort der nächstjährigen Versammlung wird Trier gewählt.

Hierauf wird die wissenschaftliche Sitzung fortgesetzt.

X1. Herr Panse-Dresden: Referat äber Hörprüfungen.

P. erklärt das beifolgend wiedergegebene Schema für die Hörprüfung, das von der vor 5 Jahren ernannten Commission zur Berathung einer einheitlichen Form der Hörprüfung (bestehend aus den Herren Barth, Bezold, Dennert, Panse, Schwabach) aufgestellt worden ist.

$$
\begin{aligned}
& R^{c S} L \\
& c^{4} L \\
& c L \\
& \text { c } W \\
& \text { Fl } \\
& \text { St } \\
& C_{2} C_{1} C_{C} C_{2} C_{1} C e
\end{aligned}
$$

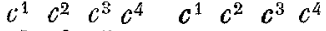

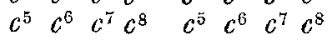

$\mathrm{Fl}=$ Flüstern, $\mathrm{St}=$ Stimme, $\mathrm{cS}=\mathrm{c}$ vom Scheitel, ein Strich von C S nach $R$ oder $L$ bedeutet: $c$ wird vom Scheitel nach rechts oder links lateralisirt, $\mathrm{c}^{4} \mathrm{~L}=\mathrm{c}^{4}$ in Luftleitung, $\mathrm{cW}=\mathrm{c}$ von der Warze. Die rechts oder links nicht gehörten $\mathrm{C}\left(\mathrm{C}^{2}-\mathrm{c}^{\mathrm{s}}\right)$ werden gestrichen. Also schreibt man auch nicht mehr: Galtonpfeife, sondern die Tonhöhe in entsprechendem $c$. Von den 5 Herren der Commission waren 4 dafür, dass beim Flüstern mit Zahlen geprüft werde. Die Hördauer ist mit $\mathbf{c}$ und $\mathrm{c}^{4}$ zu prüfen (anzugeben, ob normal oder $\mp \mathrm{x}$ Sekunden). Der Rinnesche Versuch wird von 4 der 5 Herren für überflüssig gehalten. Die Prüfung mit der continuirlichen Tonreihe wird nur für besondere Fălie als nothwendig erachtet. Die Entfernung, in der die Sprache verstanden wird, ist in Metern anzugeben. Es empflehlt sich für Obrenärzte die Anschaffung eines Clichés mit obigem Schema.

XII. Herr Denker-Hagen: Das Monotremenohr in phylogenetischer Beziehung.

Die yom Prager Zoologen Six ta aufgestellte Behauptung, dass den Monotremen ein Os quadratum zukomme, ist nach Den ker's Untersuchungen unrichtig. Denker hält in Uebereinstimmung mit van Băumelen bei Ornithorrhynchus die. Gelenkfäche des Unterkiefers, die Sixta als Os quadratum in Anspruch nimmt, für die untere Fläche des Os squamosum, und das bei Echidna als Os quadratum von Sixta aufgefasste, hinter dem Cavum tympani gelegene Knochenstück für einen Theil des Os mastoideum. Weiterhin fand Denker im Gegensatz zu Sixta, dass das Vorhofsfenster der Cloakenthiere ausschliesslich vom Os petrosum umgrenzt wird. Eine Naht zwischen Petrosum und Occipitale laterale hat Denker nicht gefunden. Im Gegensatz zu den Sauriern ist die knöcherne Labyrinthkapsel der Monotremen von einem einheitlichen Knochen, dem Os petrosum, umschlossen. Die MonotremenCochlea ist die am wenigsten aufgewundene unter allen Săugethierschnecken; sie ähnelt in ihrer äusseren Gestalt der Saurier-Cochlea. Die Monotremen besitzen eine allen anderen Säugethieren fehlende Papilla lagenae. Durch die nach Pritchard's Untersuchungen mit Cortischen Pfeilern und Tunneln 
versehene Macula acustica wird eine gewisse Aehnlichkeit mit der Säugerschnecke erzeugt. Eine Lamina spiralis secundaria fand Denker nur bei Echidna, nicht bei Ornithorrhynchus. Bei diesen beiden Monotremen-Repräsentanten fehlt der knöcherne äussere Gehörgang. Ihre Ohrmuscheln, deren Vorhandensein früher geleugnet wurde, baben die denkbar einfachste Form. Das Trommelfell besteht im Gegensatz zu dem Sauriertrommelfell, dem die Membrana propria fehlt, aus den bekannten 3 Schichten. Die Pars Haccida, die bei Eichidna aus musculären Elementen besteht, ist von der Pars tensa getrennt. Es besteht ein mit dem Os petrosum verbundener, bindegewebiger Annulus tympanicus. Hammer und Amboss sind durch Syndesmose so fest verbunden, dass sie nur als ein Ganzes functiouiren können. Stapes fehlt. Dafür findet sich eine Columella, die bindegewebig, resp. Knorpelig im Vorhoffenster befestigt ist. Statt des Hohlraumes der Pauke tindet sich bei Echidna nur eine flache, grubige Vertiefung. Bei Ornithorrhynchus fehlt in der Pauke selbst die knöcherne Umrandung der medialen Paukenwand. Beiden Vertretern der Monotremen fehlt der Musc. stapedius, dagegen besitzen beide wohlausgebildete Musc. tensores tympani, die als ein Attribut des Săugethierohres anzusehen sind. Bei Ornithorrhynchus besteht statt der Tuba eine breite Communication zwischen Rachen und Pauke, bei Echidna dagegen existirt eine knorpelig-häutige Röhre. Eine Crista vestibuli fand Denker nur bei Echidna. Im Vorbof fand Denker nur 4 Foramina für die Aufnahme der Bogengänge. Letztere erinnern im Bau an die Bogengănge der Mammaria. Eine Fossa subarcuata fand sich nur bei Ornithorrhynchus, ein Meatus acusticus internus nur bei Echidna. Nach alledem zeigt das Monotremenohr bei makroskopischer Betrachtung mancherlei Anklänge an das Ohr der Saurier, steht aber bei näherer Betrachtung dem Säugethierohre näher als dem Reptilohr. Es stellt eine Uebergangsform $z$ wischen beiden dar.

\section{Discussion: Herr Berthold-Königsberg.}

XIII. Herr Bönninghaus-Breslau: Zur Anatomie und Physiologie des Walohres.

Da die Entwicklungsgeschichte eine kurze Wiederholung der Stammesgeschichte ist, ist anzunehmen, dass die Vorfahren der Wale Landsăugethiere waren. Die daraus sich ergebende Frage, welche Veränderungen das Walohr durch Anpassung an das Wasserleben anatomisch und physiologisch erlitten hat, hat Bönninghaus durch Ohruntersuchungen eines Zahnwales, Phocaena communis, des Braunfisches, studirt. Die Resultate der B ö nainghaus'schen Untersuchungen, die durch eine Reibe guter Zeichnungen während des Vortrages anschaulich gemacht und nach dem Vortrage näheren Interessenten am Präparate demonstrirt wurden, sind folgende: Die Ohrmuschel fehlt bei Phocaena wie bei allen Walen. Der Gehörgang ist fast obliterirt, die Gehörgangsmuskeln sind rudimentär und homolog denen des Seehundes. Der hintere Nasenraum gehört nicht zur Nase (i. e. Spritzsack), sondern ist der Nasenrachenraum; denn in ihm liegt die Rachenöffrung der Tube mit der Tubenmusculatur, deren Existenz bisher geleugnet wurde. Die letztere besteht wie bei den Landsăugern aus dem Levator veli palatini und Dilatator tubae. Letzterer offfnet nur die Rachenöffnung der Tube, während die übrige Tuba auch in der Ruhe durch Wandspannung seitens der Nachbartheile klafft. Die Paukenventilation findet nicht beim Schlucken, sondern beim Inspirationsact statt, wie die anatomische Anordnung des Dilatator beweist. Die Zweckmässigkeit dieser Anordnung wird klar, wenn man bedenlet, dass das Thier beim Herunterschlucken seiner Nabrung sich meist Beute suchend mit der Nase unter Wasser befindet. Vom Mittelohre aus haben sich an der Schädelbasis weit ausgedehnte Lufträume entwickelt, die ihre Wandspannung auch durch die Nachbartheile erhalten. Sie haben nach Bönninghaus den Zweck, den Kopf des Thieres gegenüber dem übrigen Körper derart $z n$ erleichtern, dass das Thier in Ruhelage schräg mit dem Kopf nach oben liegt, wobei das Spritzloch sich über Wasser befindet, und dadurch ungehindert atbmend, der nötigen Ruhe pflegen kann. Das ansgedehnte, merkwürdig angeordnete Venensystem des Mittelohres hat keine specifische Bedeutung für das Ohr. Es ist nur als Theil des ganzen Venensystems zu betrachten, das dazu dient, die grosse Menge Blut 
zu beherbergen, über das die Wassersäuger offenbar zu dem Zwecke verfügen, dass sie an der Oberfläche des Wassers liegend durch wenige Atemzüge mittels der grossen Lungen möglichst viel Blut oxydiren können. Dadurch kann das quasi in den Zustand der Apnoë versetzte Thier lange Zeit tauchen. Da das Thier mit dem Ohre unterhalb der Wasserlinie schwimmt, verzichtet es auf die Schallübertragung durch Luftleitung. Dieser Weg wäre auch für das Hören wenig brauchbar, da der Stapes ankylotisch ist. Da das Thier ein gut ausgearbeitetes Labyrinth aufweist und, wie die Walfischjäger wohl wissen, ein feines Gehör besitzt, muss der Schall dem Thiere durch Knochenleitung zugeführt werden. Der Hamulus laminae spiralis, dessen Existenz geleugnet wurde, ist bei dem Thiere vorhanden, also auch das Helicotrema, dessen angebliches Fehlen für die Vorstellung von der Fortbewegung der Perilymphe von Bedeutung war. Nach alledem ergiebt sich, dass nur das innere $\mathrm{Ohr}$ beim Wale seine alte physiologische Bestimmung behalten hat. Dass änssere Ohr ist völlig rudimentär, das weithin ausgebaute mittlere $\mathrm{Ohr}$ hat einen Functionswechsel erlitten: es dient theils der Unterbringung venösen Blutes, theils - hauptsächlich - einem statischen Momente.

\section{Discussion:}

Herr Denker-Magdeburg: D enker hat sich auch mit der Anatomie der Wale und speciell des Braunfisches eingehend befasst. Das Corpus cavernosum ist ihm auch beim Seehund bekannt.

Hierauf tritt eine halbstündige Frühstückspause ein.

XIV. Herr Biehl-Wien: Der Verlauf des Vorhofnerven im Hirnstamme.

Der Schnecken- und der Vorhofsast sind anatomisch und physiologiseh völlig von einander gesonderte Gebilde. Deshalb ist die Ewald'sche Bezeichnung des Nervus acusticus als Nervus octavus eine berechtigte. Für die Behauptung, dass dem Schneckenast die medialen, dem Vorhofsast die lateralen Fasern zukommen, fehlen noch die Beweise. Experimentelle Verletzungen betrafen meist beide Aeste. Biehl hat an Schafen und Pferden Versuche gemacht, deren Ergebnisse er im vorigen Jahre der Wiener Akademie der Wissenschaften vorgelegt hat. Die Vorsuche an Schafen wurden fortgesetzt, die Versuche an Pferden als zu schwierig aufgegeben. Es folgt eine genaue Schilderung der Methodik der Bichl'schen Versuche und deren Ergebnisse.

Die demonstrirten Marchi-Präparate liessen erkennen: derbe, schwarze Schollen zwängen sich zwischen spinale Trigeminuswurzel und Corpus rectiforme. Sie ziehen zum dreieckigen und grosszelligen Kern. Unter letzterem ist die spinale Acusticuswurzel, der Deiters'sche und Bechterew'sche Kern zu verstehen. An den Präparaten des Falles VIII war die Kleinhirnbahn an der medialen Seite des Pedunculus cerebelli - lateral vom Bindearm - - schön zu sehen.

Durch Biehl's Befunde ist es zur Thatsa che erhoben, dass der mediale Antheil des Nervus octavus die Fasern des Ramus vesti bularis darstellt.

XV. Herr Dennert-Berlin: Acustische Untersuchungen über Mittönen und die Helmhoitz'sche Lehrevon den Tonempfin dungen.

D. hat den Werth der Helmholtz'schen Resonanztheorie einer experimentellen Prüfung unterzogen. D. prăcisirt zunächst den Unterschied in der Erregung zum Mittönen bei Körpern von gleicher oder nahezu gleicher Abstimmung, also von gleicher oder nahezu gleicher specifischer Erregbarkeit und bei Körpern, denen solche Beziehung fehlt. Die ersteren Körper erregen sich durch relativ geringe Schallkräfte zu sehr intensivem Mittönen und übertragen leichter, intensiver als die anderen Körper und vor allem in demselben Sinne die Erregung auf Körper von gleicher specifischer Erregbarkeit, die mit ihnen ein Ganzes bilden oder als Ganzes mit ihnen schwingen. Dieser experimentell leicht festzustellende Unterschied ist bei der Nachprüfung der Helmholtz'schen Lehre streng zu beachten. Wichtig ist ferner bei der Frage des Mittönens, ob die betreffenden Körper in demselben oder in verschiedenen Medien sich befinden. In gleichen Medien erregen sich die 
Körper, wofern sie nur von gleicher oder nahezu gleicher Abstimmung sind, sehr leicht zum Mittönen. Sebr schwer gelingt aber die Erregung zum Mittönen, wenn sich die Körper in ungleichen Medien befinden. Die Angaben über den Einfluss des Wassers auf die Schwingungsverhältnisse in ihm schwingender Körper sind bisher sehr vage und weit anseinandergehende gewesen. Danach wird $z$. B. der Ton einer Stimmgabel im Wasser um $11 / 2$ bis 2 Tonstufen tiefer. Es ergab sich das Gesetz, dass Körper ceteris paribus sich in gleichen Medien am besten zum Mittönen erregen, wenn sie von gleicher Abstimmung, in verschiedenen Medien, wenn sie von adäquater Abstimmung sind, d. b. wenn der erregende, unter dem Einfluss des einen Mediums stehende Körper mit derselben Schwingungszahl schwingt, wie der andere, zu erregende, unter der Einwirkung des anderen Mediums stehende Körper. Die Erregung einer in Flissigkeit befindlichen Stimmgabel durch eine in der Luft befindliche konnte objectiv überzeugend nachgewiesen werden und zwar bei fester Leitung (analog der Knocbenleitung). Die Versuche ergaben, dass zur Erregung ron Resonatoren in fester Leitung ein äusserer Hilfsap parat nicht erforderlich ist. Sehr viel schwerer ist eine Erregung in Flussigkeit befindlicher Resonatoren bei Luftleitung, weil die Schallbewegungen der Luft sehr schwer auf Flüssigkeiten übergehen. Es musste also eine Versuchsanorduung gefunden werden, bei der die Schallwellen der Luft besser auf Flüssigkeiten und in ihnen enthaltene Resonatoren übertragen wurden, um eine Analogie mit dem Ohre zu schafien. Durch die Verwerthung der oben angegebenen, von $D$, gefundenen physikalischen Thatsachen gelang es eine einfache, dem Paukenhöhlen. me chan is mus an a log e Versuchsanordnung herzustellen. Diese Thatsache spricht nach D. für den Werth der Helmboltz'schen Hypothese, zumal die Resonatoren durch geringe Schallkrätte erregbar seien und durch erstere die Erregung leicht, intensiv und in demselben Sinne auf Körper von gleicher specifscher Erregbarkeit übertragen würde, was für die Frage der qualitativen Schallübertragung auf die Endansbreitung des Acusticus im Labyrinth von Wichtigkeit sei. Die acustischen Untersuchungen D.'s ergaben ferner, dass zur besseren Schallübertragung auf in Flüsigkeiten befindliche Resonatoren in Luftleitung analog der Hörprüfung in Luft leitung und dem Hören überhaupt ein äusserer Hilfsapparat sebr $\mathrm{z}$ weck mässig ist, während er, wie erwäbnt, bei Uebertragung in Knochenleitlug aicht erforderlich erscheint.

Discussion:

Herr Kayser-Breslau: Die Dennert'schen Versucbe haben uns in der Lösung des Problems, wie in der Luft erregte Schallschwingungen sich auf einen im Wasser befindlichen Resonator übertragen, einen Schritt weiter geführt. Aber in ihrem Kernpunkt ist diese Frage durch die D'schen Versuche noch nicht gelöst worden. Bei der D.'schen Versuchsanordnung werden nämlich die Schwingungen der in der Luft befindlichen Stimmgabel, nachdem sie indirect in Mitschwingungen versetzt ist, auf die im Wasser befindliche Gabel dureb directe, feste Ferbindung übertragen (von Stiel zu Stiel odor durch dazwischengeschaltete Columella). Es fehlt also bei der Versuchsanordnung die Analogie mit dem Ohre, bei dem sich doch zwischen Stapesplatte und Membrana basilaris ein flüs siges Medium, die Perilymphe, befindet. Gerade bei der Uebertragung von Schallschwingungen aus der Luft oder aus einem festen auf ein flüssiges Medium findet nun aber, wie wir wissen, eine starke Abschwächung der Schallschwingungen statt und die Frage ist, wieso das Ohr trotz dieser Schallabschwächung so leistungsfähig ist.

Herr Sc h miedt - Leipzig-Plagwitz: Die Dennert'sche Versuchsanordnung entspreche wohl doch den Verhältnissen im Ohre.

Herr Zimmermann-Dresden: Entsprechend Joh. Müllers Versuchen wird die Knöchelchenkette noch immer als Columella aufgefasst. Z. erinnert an eine Arbeit ans Fixners Institute. Die Kette der Ossicula kann den Schall nicht übertragen: sie dient nur zur Dämpfung des Schalls.

Herr Panse-Dresden: Die Versuche, auf die Herr Zimmermann aufmerksam machte, sind mit Vorsicht aufzunehmen. 
Herr Dennert-Berlin: D. giebt zu, vom Ziele noch entfernt zu sein, freut sich aber, in der Lösung der Frage schon so weit gekommen zu sein.

XVI. Herr Panse-Dresden. Wo entsteht der Ohrschwindel? P. setzt auseinander, auf welchen Nervenbahnen wir über unser Verhältniss im Raume unterrichtet, wie wir darüber getäuscht und schwindlig werden. Der Vortrag wird anderweitig veröffentlicht werden.

\section{Discussion.}

Herr Berthold-Königsberg, B. berichtet über einen Patienten mit nach seiner Meinung wohl operativ entstandenem Schwindel, der ihn auf der Strasse, als ihn B. ansprach, beschimpfte.

Herr Thost-Hamburg: Das Thema des Ohrschwindels sei anf die Tagesordnung für die diesjährige Naturforscherversammlung in Hamburg gesetzt worden, bei der die otologische und die laryngologische Section vereinigt sein würden. Th. ladet zu zahlreichem Besuch in Hamburg ein.

XVII. Herr Berthold-Königsberg: Ueber ent otische Tön e. Entotische Gerăusche setzen gewöhnlich einen pathologischen Zustand im Ohre voraus. B. selbst, dessen Ohren bis 1884 gesund waren, acquirirte damals einen Rachen- und Tubenkatarrh mit Autophonie. Darauf stellte sich links 1. ein hohes $K$ lingen $\left(=f_{3}\right)$ ein, das indes auch bei Ohrgesunden vorkommt, 2. ein in der Stille auftretendes Geräusch ron siedendem Wasser, 3. ein glockenhelles $c_{3}$, das nur bei schüttelnden Kopfbewegungen auftrat, mit Aufhören derselben sofort sistirte. Bei theilweisem Verschluss des Gehörganges gelangt der Ton $c_{3}$ durch kleine Trommelfellerschütterungen nicht zur Wahrnehmung, wohl aber bei vollständigem Verschluss. Beim Gehen nach angestrengter Arbeit trat auch der Ton $c_{3}$ auf und zwar synchron mit dem Pulse. Diese Tonerscheinung beruht wohl nach B. darauf, dass die Ossicula durch geringe Lockerung ibrer Gelenkverbindung in Bewegung geraten. Bei starker Lockerung würde ein Geräusch statt des Tones auttreten. Das Auftreten von $c_{s}$ bei geistiger Arbeit glaubt B. auf die Hyperämie beziehen zu sollen, die nach angestrengter Geistesarbeit im Kopfe sich einstellt.

\section{Discussion.}

Herr Schmeden-Oldenburg: Wenn das Tönen im Ohre theilweise auf Hyperămie beruhe, so könne therapeutisch versucht werden, einen Eisbeutel aut den Hals zu legen, der die Temperatur im Gehörgang herabsetze.

Herr Berthold: B. will diesen therapeutischen Vorschlag wegen gewisser Bedenken an sich nicht erproben.

XVIII. Herr Habermann-Graz: Zur Entstehung der Taubs tummheit. Herr $H$. begnügt sich mit Demonstrationen von Mikrophotogrammen der btr. Präparate. Im ersten Falle handelt es sich um einen 73 jährigen Taubstummen, der links Perforation und Verkalkung, rechts Residuen anfwies. Stapes durch Hyperostose des Knochens und bindegewebig fixirt. Rundes Fenster knöchern verschlossen. Ambos mit Hammerhropf bindegewebig an der lateralen Atticuswand fixirt. Die Taubstummheit ist wohl hier also - soweit cerebrale Ursachen anszuschliessen sind -.. wesentlich durch Verwachsung des runden Fensters und Fixation des Steigbügels im ovalen Fenster bedingt gewesen. Im Falle 2 bandelte es sich um Hyperostose der Labyrinthkapsel bei einem erwachsenen Patienten. Reste des Gehörs waren bei dem Patienten noch vorhanden. Das ovale Fenster war frei, das runde durch Verknöcherung verwachsen. Der zweite Fall beweist, dass, wenn auch das runde Fenster knöchern verschlossen ist, Gehör vorhanden sein kann; wenn nur das ovale Fenster für das Ausweichen der Schwingungen der Labyrinthflüssigkeit zugängig ist.

XIX. Herr Scheibe-München: Zur Ostitis der Labyrinthkapsel. Die die Grundlage der Sklerose bildende Ostitis der Labyrinthkapsel ist abgesehen von kleinen isolirten Stellen über der Knochenerkrankung gewöhnlich nicht mit nachweisbaren Veränderungen der Mittelohrschleimhaut vergesellschaftet. Darin decken sich Sch's Untersuchungsresultate mit denen von Politzer und Siebenmann. Nur Habermann vertritt auf Grund seiner Untersuchungsergebnisse die Ansicht, dass in diesen 
Fällen die nur anscheinend normal gewordene Scbleimbaut in einem früheren Stadium entzündet war. Sch. berichtet über 2 eigene nene Untersucbungen: Im ersten Falle handelt es sich um einen 3 Wochen ante exitum plötzlich ertaubten 20 jăhrigen Mann mit chronischer Mittelobreiterung und Cholesteatom, das vermittelst einer Bogengangsfistel dem Eiter den Eingang ins innere Obr vermittelt hatte. Die Schläfenbeinuntersuchung ergab am Bogengangswulst im Aditus, an der Aussenfläche der Labyrinthkapsel, an dea Schenkeln und der Fussplatte des Stapes und im ovalen Fenster neugebildete Knochenbalken. In der Paukenwand unterhalb des Facialis fand sich ein grosser mit Narbengewebe erfüllter Defect im Knochen. Die weiteren Veränderungen werden an vergrösserten Zeichnungen demonstrirt: in der Labyrinthkapsel fand sich oberhalb des ovalen Fensters ein stecknadelkopfgrosser Knochenherd mit osteoidem, unverkalktem Gewebe zwischen dea Knochenbälkchen. Die Oberfläche dieses Knochenkerns ist cariös ausgefressen. Der Knorpelbelag zeigt sich am ovalen Fenster ganz geschwunden, am Stapes vorhanden. Es besteht also hier 1. Osteosklerose, bei der der Knochen auf Kosten des Markes zugenommen hat und nebenbei 2. ein Herd, bei dem das Markgewebe auf Kosten des Knochens zugenommen hat, Der letztere Herd stellt einen bei chronischer Ostitis, bei der in der Regel Osteosklerose bestebt, ungewöhnlichen Vorgang dar. Die Localisation entspricht hier der Angabe Siebenmann's, nach dem die Spongiosirung der Labyrinthkapsel ihren Lieblingssitz oberhalb des ovalen Fensters hat. Habermann fand solche Knochenherde bei chronischen Mittelohreiterungen und Residuen. Auch hier in Sch.'s Fall ist wohl die chronische Otitis media purulenta ätiologisch in Anspruch zu nehmen. Vielleicht ist in einem Theil der Fälle von abgelaufener chronischer Mittelohreiterung an der nachträglich eintretenden bedeutenden Hörverschlechterung das Auftreten solcher Knochenherde in der Labprinthkapsel schuld.

Der zweite, bereits in der Zeitschr. f. Obrheilk. Bd. 27 veröffentlichte Fall betrifft einen $81 / 2$ jährigen, an Scharlachdiphtherie verstorbenen Taubstummen. Residuen von Otitis interna: viel neugebildetes Bindegewebe am Knochen. In der Labyrinthkapsel ist offenbar Knochen neu gebildet und wieder zerstört worden. Im Promontorium unterbalb des ovalen Fensters ein Herd mit Kuochenauftreibung nach der Pauke hin und mit erweiterten Markräumen. Der neugebildete, grösstentheils schon verkalkte Knochen zeigt nur an einzelnen Stellen noch osteoïde Substanz. Der Knorpelbelag des Vorboffensters ist intact. Durch die frische Scharlachotitis kann die Knochenerkrankung im Promontorium nicht bedingt sein. Ob die Ostitis in der Schneckenkapsel mit Gefässveränderungen in der Pauke oder mit der Otitis interna zusammenbängt, steht dahin. Scheibe's 2 Fälle bestätigen die Behauptung Habermann's, dass dieselbe Knochenerkrankung wie bei Sklerose des Mittelohres, auch bei chronischer Mittelohreiterung [vielleicht auch bei Otitis interna] eintreten kann.

Discussion:

Herr Habermann-Graz: Habermann freut sich über die Bestätigung seiner vor 10 Jahren veröffentlichten und bisher stets todtgeschwiegenen Befunde. Habermann berichtet über die Schläfenbeinbefunde bei einer $28 \mathrm{jäh}$ rigen, seit der Jugend an eclamptischen Krämpfen leidenden Idiotin. Er fand rechts einen Knochenherd unterhalb der runden Fenstermembran, links einen in die Fensternische und in die äussere Wand der Schneckenkapsel hineingehenden Herd, Periostitis und Hyperämie am Promontorium sowie neben den Knochenveränderungen deutliche chronisch-entzündliche Schleimhautveränderungen. Habermann glaubt, dass die Ostitis chronica von einer Entzündung der Pauke ausgeht. Habermaun fand die Knochenheerde fast regelmässig unmittelbar am ovalen Fenster, meist dicht vor dem Steigbügel. Auch um die Bogengänge herum und im inneren Gehörgange kommen solche Herde vor. Habermann demonstrirt entsprechende Präparate.

Herr Scheibe-München: Die Ostitis bei Sklerose kann man nicht mit Schleimhautentzündung in genetischen Zusammenhang bringen. Denn einmal bestehe die Sklerose meist doppelseitig und zweitens fehlten dabei entzündliche Erscheinungen an der Schlei mbaut. 
Herr Panse-Dresden: Das constitutionelle Moment ist bei diesen Fällen von Spongiosirung nicht zu vernachlässigen, da ja die letztere Aehnlichkeit mit der Osteomalacie habe und diese Fälle sich gerade nicht selten an das Puerperium anschliessen.

Herr Habermann-Graz: Habermann versteht hier unter Mittelohrentzündung h is tolog is ch entzündlich veränderte Schleimbaut. Hab erman glaubt bestimmt, dass die Erkrankung vom Mittelobr ausgeht. Weun frühere Untersucher, wie Herr Scheibe erwähnte, die Mittelohrschleimhaut normal fanden, so mag das für manche Fälle stimmen. In der Umgebung des Herdes aber werden immer histologische Verănderungen gefunden.

XX. Herr Görke-Breslau: Ueber die Caries der Gehörknöchelchen.

Patbologisch-anatomische Untersuchungen über Caries der Ossicula wurden bisher nur wenig gemacht. Die meist nur operativ gewonnenen Erfahrungen sind zu einseitig, um über das Wesen der Erkrankung einen sicheren Aufschluss zu geben. Bei den operativen Beobachtungen handelt es sich meist um fortgeschrittene Fälle, nur sehr seiten um Anfangsformen, deren Studium zur Aufklärung der Genese gerade wichtig erscheint. Auch über die reparativen Vorgänge und Spontanheilungen bei Kuöchelchencaries können klinische Erfahrungen wenig oder keincn Aufschluss geben, ebensowenig über die Beziehungen der Erkrankung zu den Erkrankungen der übrigen Theile des Obres, namentlich der Mittelohrwände und über ihr gegenseitiges $\mathrm{Ab}$ hängigkeitsverbältniss. Die von G örke gefundenen Ergebnisse von $800 \mathrm{Ob}$ ductionen im Breslauer Allerheiligen-Hospital sind folgende: (Görke bemerkt vorher, dass stets auch mikroskopisch untersucht wurde, da bei makroskopischer Betrachtung allein durch Rinuen, Zerklüftungen an der Oberfläche der Ossicula, die die Folge obelflächlicher Eintrocknung darstellen, cariöse Usuren $a . s, w$, vorgetäuscht werden können. Bei der am besten an Serienschnitten auszufübrenden mikroskopischen Untersuchung ist zu beachten, dass der Befund lacunärer Recorption allein die Diagnose der Caries nicht gestattet, weil besonders im jugendlichen Knochen auch physiologisch sich fortwäbrende Knochenauflösungs - und Appositionsprocesse abspielen). Die cariöse Knöchelchenerkrankung beginnt gewöhnlich mit einer Periostitis. Durch Fortplanzung des Processes durch die Havers'schen Canäle kommt es dann secundär zur Erkrankung des Endosts und des Marks. Allein das umgekehrte Verbalten ist nicht so selten, wie allgcmein angenommen wird; denn es wurde bei frischer Mittelohrentzündung wiederholt eine Betheiligung des Marks constatirt, ohne dass das Periost überhaupt oder wesentlich befallen war. In solchen Fällen, bei deneu die Entzündung offenbar dircet auf dem Lymphwege sich ins Mark fortpflanzt, kann es sogar zur Nekrose des Markes, zur Bildung centraler Abscesse bei intactem Periost kommen, welch' letzteres hierbei häufig durch stark sklerotisch verdickte Knochenmasse vom medullären Herde getrennt ist. Diese ostromyelitiscben Herde können ihrerseits an die Oberfiäche durchbrechen und zur Fistelbildung führen. Die Periostitis kann sich entweder durch die $\mathrm{Havers}$ 'schen Canäle nach innen fortpflanzen oder es kann bei heftiger Periostentzündung und fortbestehender Mittelohreiterung die Knocheneinschmelzung direkt nach dem Centrum fortschreiten, bis das Mark - bäufig in grosser Ausdehnung - freiliegt. Was die Frage der Spontanheilung, deren Möglichkeit durch klinische Beobachtungen von Gompertz, Scheibe, Kretschmann, Hessler sichergestellt ist, anlangt, so sind bisweilen schon makroskopisch an gewissen, mit glatter Schleimbaut überzogenen Deformitäten abgeheilte Krankheitsprocesse zu erkennen. Bei der Ausheilung zeigt sich entweder ein glatter, ron gesunder Schleimbaut überzogener Defekt oder ein durch Narbengewebe erfüllter Defect oder hyperostotische Verdickungen oder Exostosenbildung durch Knochenneubildung am Ort der Heilung. Spielt sich gleichzeitig derselbe Process an den benachbarten Paukenwänden ab, so kommt es leicht zur Fixirung der Ossicula, am bäufigsten zur Verwachsung des Hammerkopfes mit dem Tegmen tympani. Auch die meduilären Herde können, wenn es nicht zur Sequesterbildung gekommen ist oder wenn der gebildete Sequester bereits ausgestossen wurde, ausheilen und zwar unter Bildung von Narbengewebe und unter Knochen- 
neubildung vom Endost aus, woraus eine partielle oder totale Sklerosirung des Knöchelchens mit völligem Verschwinden des Markraumes resultirt. Bezüglich der Localisation der Caries wurden die alten Angaben bestätigt gefunden, dass der Ambos hăufiger, als der Hammer, am hăufigsten der lange Ambosschenkel, der Kopf des Hammers häufiger als der Griff erkranken. Aber auch Theile, deren Ergriffensein als selten gilt, fanden sich oft mitbetheiligt, ja bisweilen allein befallen z. B. der Hammerhals und die allerdings am widerstandsfähigsten Gelenkflächen. Auch isolirte Caries des Amboszörpers wurde wiederholt constatirt. Bezüglich des Rückschlusses, den der otoskopische Befund anf den Sitz der Erkrankung gestattet, hat in letzter Zeit Leutert ein Schema aufgestellt, in dem der Lage der Perforation eine hohe diagnostische, bisweilen pathognomonische Bedeutung zugeschrieben ist. Es zeigte sich nun bei den Obductionsergebnissen, dass eine Reihe von Fällen in dieses Schema nicht hineinpassen. Am constantesten zeigte sich noch der Zusammenhang von Perforation der Shrapnell'schen Membran mit Erkrankung des Hammerkopfes und von Perforation hinten oben mit Amboscaries. Aber in Fällen, wo sogar mikroskopisch Amboscaries nachgewiesen war, fehlte bisweilen jede Perforation. (Diese Fälle betrafen stets tuberculöse Kinder.) Andrerseits war in manchen Fällen mit grossem, mitunter randständigem Defect ausser geringer Verdickung der periostalen Bedeckungsschicht nichts Pathologisches an den Knöchelchen nachzuweisen. Ein constanter Befund war der, dass, wenn der Knochen am Tegmen, an der äusseren Atticusoder hinteren Gehörgangswand cariös war, Knöchelchencaries nie vermisst wurde. Die Ossicula scheinen also, wenn es überhaupt zu einer Betheiligung des Knochens kommt, als die am wenigsten widerstandsfähigen Theile zuerst ergriffen zu werden. Jedenfalls geht aus diesen Sectionsbefunden hervor, dass man die Diagnose auf dem otoskopischen Befunde allein nicht immer aufbauen darf und dass die Erfahrungen der pathologischen Anatomie diejenigen der Klinik hier za ergänzen haben.

\section{Discussion:}

Herr Kretschmaın-Magdeburg: K. freut sich über die Bestätigung seiner Ansicht, dass Ort und Lage der Perforation keinen erheblichen Rückschluss auf Art und Ausdehnung der Knöchelchenerkrankung gestatten. K. fragt an, ob bei den Fällen mit seltener Localisation (am Hammerhals etc.), die nach seinen E'rfahrungen auf Tuberculose hinweisen, letatere vorlag.

Herr Ehrenfried-Berlin: E. freut sich über die gefundenen Resultate, die einen Fortschritt für die Würdigung der conservativen Behandlung chronischer Mittelohreiterungen involviren.

Herr Biehl-Wien: Die Bestimmung in Oesterreich, dass das Bestehen von Perforationen Dienstuntauglichkeit bedinge, soll in diesem Jahre beseitigt werden und B. ist von der österreichischen Regierung mit der Bearbeitung dieser Frage betraut worden. Es war ihm interessant und wichtig zu hören, dass er sich auf das Leutert'sche Schema nicht ohne Weiteres verlassen könne.

Herr Görke: Da das Sectionsmaterial zum grossen Theil von Phthisikern stammt, so lag wahrscheinlich in jenen Fällen mit seitener Localisation der Caries hăufig Tuberculose vor. Herrn Ehrenfried sind seine therapeutischen Folgerungen zuzugeben, denn die Heilbarkeit der cariösen Erkrankungen ist hier anatomisch festgestellt worden.

XXI. Herr Deutschländer. Demonstration von 4 Fällen von typischem Rhinosklerom. In 2 Fällen war besonders die Nase, in 2 Fällen besonders der Larynx (starke subglottische Wülste) befallen.

XXIl. Herr Hinsberg-Breslau: Ueber den Infectionsmechanismus bei Meningitis nach Stirnhöhleneiterug.

Während bei chroni schen Stirnhöhleneiterungen, die zur intracraniellen Complication führen, gewöhnlich ein Defect der hinteren Stirnhöhlenwand besteht, fehlt in acuten Fällen gewöhnlich jede Knochenveränderang. Manchmal wird eine solche auch bei chronischen Făllen vermisst. Als der Infectionsweg werden in solchen Fällen die Vasa perforantia angesehen. H. berichtet über einen Fall mit Eiterung in fast sämmtlichen 
Nebenhöhlen. Es wurde zunächst die rechte Kieferböhle, bald darauf die rechte Stirnhöhle eröffnet. Letztere enthielt viel Eiter. Die usurirte orbitale Wand wurde resecirt. Cerebrale Wand intact. Exitus 36 Stunden post operat. Die Section ergab Meningitis, besonders rechts. Dura mit Fibrinschicht belegt, zeigt über dem Stirnböhlendache einige minimale Knötchen. Knochen dünn, aber makroskopisch nicht verändert. Die genaue Knochenuntersuchung ergab das Vorhandensein zahlreicher Vasa perforantia. In einigen Gefässen alte, zum Theil organisirte Thromben. Die Duraherde bestehen aus abgekapselten Rundzellen mit massenhaften Bacterien. Dura selbst an diesen Stellen stark verdickt.

\section{Discussion:}

Herr Habermann-Graz: H. berichtet über einen sehr merkwürdigen Fall von Stiruböhlenempyem, der zur Autopsie kam. Durch dieselbe wurde festgestellt, dass es sich um einen von einer Perityphlitis ausgehenden, embolischen Hirnabscess rechts gehandelt hatte, der secundär eine rechtsseitige Stirnhöhleneiterung ausgelöst hatte. Letztere hatte infolge Durchbruchs nach der linken Stirnhöhle in der linken Nase manifeste Empyemsymptome erzengt.

XXIII. Herr Mann-Dresden: Ueber Mucocele des Siebbeins.

Ein 39 jähriger Schlosser, der in der Jugend ein Kopftrauma erlitten und vor 20 Jahren Lues acquirirt hatte, bemerkte vor 2 Jahren ein Heraustreten des rechten Auges und Versehlechterung des Sehvermögens mit wechselnden Beschwerden. Der Augenarzt constatirte anfangs normalen Augenhintergrund, allmählich stellte sich aber Staungspupille ein und Herabsinken der Sehschărfe auf $\mathrm{S}=\frac{6}{24}$. Mann constatirte im April d. J. bei dem Pat. Folgendes: Rechter Bulbus stark nach aussen und vorn getrieben, im inneren Augenwinkel ein kirschgrosser, prall elastischer Tumor. In der Nase nach Abhebung der mittleren Muschel mit dem Killian'schen Speculum Siebbeinboden stark nach unten und medialwärts erweitert zu sehen. Schleimbaut blass. Knochen wird erst mit Sonde durchstossen, dann mit Hartmann'scher Zange gefenstert. Der Inhalt ist chocoladenfarbig, syrupdick, enthält keine Bacterien, aber soviel Cholestearin, dass dessen Reindarstellung leicht gelingt. Die Cyste sondert weiterhin kein Secret ab. Bei der Operation sinkt der Bulbus tief in die Orbita zurück, schnappt aber in den nächsten Tagen beim Schnauben in die alte Lage zurück. Das Sehvermögen bessert sich rasch zur normalen Sehschärfe. Die Staungspapille verschwindet, aber Nenritis ist noch vorhanden. Trotz der Annahme scheint auch bier eine angeborene Anlage für die Cyste vorhanden gewesen zu sein. In der Literatur sind nur 8 solche Fälle beschrieben; vor kurzer Zeit einer von Avellis im Arch. f. Lar. Dies ist der einzige Fall, bei dem ein rhinologischer Befund erhoben ist. Derselbe ähnelt in vieler Hinsicht dem vorliegenden. Der Fall gehört in das Grenzgebiet von Rhinologie und Ophthalmologie. Er beweist, wie nothwendig bei Tumoren der Orbita eine specialistische Untersuchung der Nase ist.

XXIV. Herr Wertheim-Breslau: W. demonstrirt einen 2bjährigen Patienten mit doppelseitigem Stirnhöhlenempyem. Im Januar 1899 war von $\mathrm{Gerber}$ die linke Stirnhöhle nach $\mathrm{Kuhnt}$, die linke Kieferhōhle nach $\mathrm{Coo}$ per eröffnet worden. Nach 5 Monaten musste die 3 Monate p. op. zugeheilte Stirnhöhle, da es sich um eine Scheinheilung gehandelt hatte, wieder von Gerber eröffnet werden und blieb ca. ein Vierteljahr offen. Im Oktober 1899 wurde wegen entsprechender Beschwerden in Elbing die rechte Stirnhöhle, die sich als intact erwiesen und nach 3 Wochen geschlossen haben soll, sowie die rechte Kieferhöhle eröffnet, in der sich nur wenig Schleim fand. Im December 1900 musste von W. die linke Stirnhöhle zum 3. Mal breit eröffnet werden. Es handeIte sich wieder um eine Scheinheilung mit Eiterstagnation in einer durch Granulationen abgeschiedenen Kammer. Die eroffneten vorderen Siebbeinzellen intact. Hautwunde nicht vernăht. Nach 8 Wochen Höhle fast secretfrei. Im März 1900 schwere Influenza mit Verschlimmerung in der linken Stirnhöhle und schwerem Empyem der rechten Stirn- und Kieferhöhle. Die rechte Stirnhöhle masste, da die unerträglichen 
Beschwerden und das hohe Fieber anders nicht au beseitigen waren, breit. nach $\mathrm{Kuh}$ at eröfnet werden. In derselben Narkose noch einmal gründliche Ausräumung der linken Stirnhöhle. Nach einigen Tagen musste auch die rechte Kieferhöhle, die vor einiger Zeit bei Anbohrung von der Alveole stinkenden Eiter ergeben hatte, breit nach Küster eröffnet werden. Erst jetzt liessen die excessiven Schmerzen und das hohe Fieber nach. In der rechten Stirn- und Kieferhöhle war massenhaft fötider Eiter und so schwere Schleimhautverănderungen gefunden worden, dass es sich hier zweifellos um: latente chronische Eiterungen handelte, die durch das Hinzutreten der Influenzainfection exacerbirt und manifest geworden waren. Ende April d. $J$. mussten noch die rechten vorderen Siebbeinzellen und die rechte Keilbeinhöble endonasal wegen excessiver Schmerzen an der Nasenwurzel und wegen Nasenrachenbeschwerden eröffnet werden. Beide Höhlen waren auch eitrig erkrankt. Jetzt sondert die rechte Stirnhöhle nur wenig schleimig eitriges Secret ab, ebenso die linke, die bis auf die Gegend am Ostium aasofrontale in ihrer ganzen Ausdehnung verödet ist. Das kosmetische Resultat ist ein relativ gutes. Augen intact bis auf geringe Parese beider Musc. recti superiores. W. meint, es empfehle sich bei der Kuhnt'schen Operation die Wunde gar nicht zu vernähen, um bei der Nachbehandlung alle Stellen der Höhle genügend übersehen zu können. Als Endziel der Operation sei die Verödung der Stirnhöble ins Auge zu fassen, und wenn diese erreichbar scheine, event. zu versuchen, die Stirnhöhle gegen die Nase durch Lappenaufpflanzung oder Aetzungen am Ostium nasofrontale abzuschliessen, um Reinfectionen von der Nase ber auszuscbalten.

XXV. Herr Ehrenfried-Berlin: Ueber conservative und opera. tive Behandlung der Mittelobreiterungen.

E. wendet sich gegen das viele Operiren bei acuten und ehronischen Mittelohreiterungen, indem er auf die Gefäbrlichkeit der Operation, die Unsicherheit der Heilung und die Ungewissheit bezüglich des functionellen Resultates hinweist. E. verwirft alle prophylactischen Indicationen für die Operation und erkennt nur curative an - und zwar unter ihnen nur das Bestehen absoluter Lebensgefahr (Meningitis und Pyämie), sowie eine so hochgradige Gehörgangsverengerung, dass eine Behandlung vom Meatus externus aus unmöglich ist. Jede Mittelohreiterung aber, die 4 Wochen lang zweckentsprechend conservativ behandelt wurde, ist nach $E$. bereits derart gebessert, dass gefăhrliche Complicationen ausgeschlossen sind. Caries und Cholesteatom berechtigen somit nach E. nicht zur Totalaufmeisslung, ebensowenig Labyrinth- und Facialissymptone. E. berichtet nun uber die Erfolge seiner conservativen Behandlung bei schweren acuten und cbronjschen Fällen etc. Schliesslich schildert $E$. die Art seines conservativen Verfahrens: E. träufelt mittels eines Augentropfglases eine schwache Lysollösung in das kranke Ohr, aspirirt nach 10 Minuten die Flüssigkeit aus dem Gehörgang und wiederholt dieses Verfahren nöthigenfalls $10-20$ mal und öfter, um allen in der Tiefe verborgenen Eiter zu Tage zu fördern.

\section{$\mathrm{D}$ is cussion:}

Herr Preysing-Leipzig. Ein grosser Theil der E.'schen Behauptungen erscheine ihm unhaltbar. Den als Stütze für seine Anschauungen angeführten Gründen fehle die 2wingende Beweiskraft. Eine eingehende Erörterung verdiene allerdings die Frage, ob und wann die Totalaufmeisselung bei uncomplicirten chronischen Mittelohreiterungen indicirt ist.

Die Herren Körner-Rostock und $\mathrm{K} u ̈ \mathrm{mmel}$ - Breslau verzichten wegen vorgerỉckter Stunde auf die Abhaltung der von ihnen angekündigten Vorträge.

Nachdem somit die Tagesordnung erschöpft ist, wird die Versammlung um 4 Uhr durch den Vorsitzenden geschlossen.

Mit dem Congress verbunden war eine reich beschickte und - dank den Bemühungen der Herren $\mathrm{Kümmel}$ und Hins berg - wohl gelungene Ausstellung vou Präparaten, Modellen, Apparaten, Tafeln, Abgüssen, Zeichnungen, Instrumenten etc. Bei der grossen Fülle von Objecten kann hier begreiflicherweise nur ein Theil derselben Erwähnung finden: 
Rostocker Ohrenklinik: Pathologisch-anatomische Präparate. Eine Sammlung kranker Ossicula. Zeichnungen und Tafeln betreffend die Anatomie bezw. Pathologie der Nase.

Herr Scheibe-München: Zeichnungen und Wandtafeln betr. Ankylose des Steigbügels bei Mittelohr-Sklerose.

Herr Denker-Hagen: Corrosionspräparate (nach Siebenmann) von Gehörorganen des Menschen, Affen, der Monotremen. Tafeln über vergleichende Anatomie des Säugethierohres, über die makroskopische Anatomie des Monotremenohres. Schädel vom Varanus salvator, Ornithorrhynchus paradoxus und Pteropus edulis. des Ohres.

Herr Habermann-Graz: Bilder aus der pathologischen Anatomie

Herr Biehl-Wien: Anatomische Trockenpräparate des menschlichen Felsenbeins. - Spirituspräparat eines Schädels mit ausgedehnter Sinusthrombose (Retrograder Transport, ausgehend von einem Thrombus im Sin. sigm. Cholesteatom). - Zeichnungen mikroskopischer Präparate und Spirituspräparate von Felsenbeinen des Schafes, die den getrennten Verlauf des Schnecken- und Vorhofastes des Acusticus beim Schafe demonstriren.

Herr Brühl-Berlin: 39 Tafeln betr. die normale und pathologische Anatomie des Ohres (aus Brühls "Handatlas der Ohrenhellkunde"). 4 durchsichtige Schädel in Xylol zur" Demonstration der Topographie und der Dimensionen der Nasennebenhöhlen. - Ein Diapositiv, enthaltend 4 Radiogramme betr. Nasennebenhöhlen, knöchernes Labyrinth vom Menschen und Affen, gesammten Mittelohrtractus. - 4 Tafeln betr. die Topographie des menschlichen Gehörorgans.

Ohrenabtheilung am Allerheiligen-Hospital-Breslau: Trockenpräparate betr. Nebenverletzungen bei Operationen. - Trockenpräparate betr. Entwicklung des Annulus tympanicus in den verschiedenen Altersstufen.

3 Klötze mit Lupenpräparaten betr. die normale und pathologische Anatomie des Mittelohres.

Stereoskopische Photogramme betr. Caries ossiculorum, Arrosion der Bogengänge des Facialis etc., Dehiscenzen am Facialcanal und am Paukenboden, Felsenbeinfractur, Schläfenbeintuberculose etc. - Zeichnungen mikroskopischer Präparate betr. Ohrpolypen, Veränderungen in Rachenmandeln, Caries ossiculorum etc.

Ein Ohrmikroskop (ron Zeiss - Jena gefertigt).

Röntgenbilder. - Eine stereoskopische Camera, mit der die ausgestellten stereoskopischen Photographien gefertigt sind.

Herr Panse-Dresden: 12 Bleistiftzeichnungen nach mikroskopischen Präparaten betr. Labyrintheiterung. - Mikroskopische Präparate vom Labyrinth der Hausmaus und Tanzmaus, nach welchen zwischen beiden kein Unterschied sichtbar ist.

Ohrenklinik-Breslau: Corrosionspräparate von Schläfenbeinen. Semper-Riehm'sche Schläfenbeinpräparate.

Herr Hinsberg-Breslau: Plattenmodelle betr. die Entwicklung der Nase bei Säugethieren und Menschen.

Herr Hartmann-Berlin: Serien von Diapositiven (Nasenanatomie).

Herr Alexander-Wien: Modell des Mittelohres.

Herr Berliner-Berlin: Gypsmodell rom Ohre cines Neugeborenen mit Atresie des äusseren Gehörganges und Aplasie der Ohrmuschel. Gypsmodell betr. Microtie und Aplasia meat. auditor. ext. bei einem 44 jähr. Manne. - Colorirte Wachsabgüsse betr. Othämatom.

Herr Kayser-Breslau: Physiologischer Apparat zur Untersuchung der Schallschwingungen in flüssigen Medien mittels eigenartigen Telephons.

Herr $\mathrm{S}$ ter $\mathbf{n}$-Breslau: Tonvariator (Flaschenapparat für continuirliche Tonreihe).

Herr Winkel-Göttingen: Apparat für Mikrophotographie.

Herr Ka tz-Berlin: Mikroskopische Präparate betr. hernienartige Zapfenbildung bei Otitis med. acut. pur. - Mikrosk. Präp. betr. die Epithelgebilde des Vorhofs (spez. der Crista acustica mit Cupula terminalis) und des 
282 XVI. WERTHEIM, Bericht über die 10. Versammiung zu Breslau.

Cortischen Organes (die Deiters'schen Zellen und ihre Verbrüderung mit den äusseren Corti'schen Zellen), - Mikrosk. Präp. betr. die Spongiosirung des Schläfenbeins. - Makroskopisches Präparat (durchsichtig) des Labyrinths (Osmiumsäure-Injection!), den spiraligen Verlauf des Nervus cochleae durch die Schneckenwindungen von der Radix cochleae an demonstrirend. Stereoskopische durchsichtige Präparate des mittleren und inneren Ohres (Diapositive).

Herr Betz-Heilbronn (Firma Dr. Determann, Heilbronn): Gypsmodelle der Nase und ihrer Nebenhöblen.

Herren Benninghoven und Sommer-Berlin: 6 Modelle aus Papiermaché nach Angaben von Prof. Trautmann betr. Operationen am Ohre in ihren verschiedenen Stadien. - Ohrmodelle, modellirt von Dr. Frohse-Berlin, Modell des Corti'schen Organes, aus Papiermaché.

Herr Edelmann-Mänchen: Die Bezold'sche Tonreibe mit Galtonpfeifen. - Laryngostroboscop nach Prof. Oertel. - 1 Satz Bezold'scher unbelasteter Gabeln.

An der überaus reichhaltigen und interessanten Instrumenten- und Apparateausstellung waren das Berliner Medicinische Waarenhaus sowie die Firmen Pfau und Détert-Berlin, Katsch-München, Elektrotechn. Institut Frankfurt a. M., Georg Härtel-Breslau betheiligt. 